This is the accepted version of the following article

Controlling the surface chemistry of graphene oxide: Key towards efficient ZnO-GO photocatalysis

S. Víctor-Román, E. García-Bordejé, J. Hernández-Ferrer, J.M. González-Domínguez, A. Ansón-Casaos, A.M.T. Silva, W.K. Maser, A.M. Benito

Published in Catalysis Today (C) Elsevier

The final edited and published version is available under

https://doi.org/10.1016/j.cattod.2019.05.049 


\section{Controlling the surface chemistry of graphene oxide: Key towards efficient $\mathrm{ZnO}$ - GO photocatalysts}

Sandra Víctor-Román ${ }^{a}$, Enrique García-Bordejéa , Javier Hernández-Ferrera ${ }^{a}$, José M. González-Domínguez ${ }^{\mathrm{a}}$, Alejandro Ansón-Casaos ${ }^{\mathrm{a}}$, Adrián M.T. Silva ${ }^{\mathrm{b}}$, Wolfgang K. Maser $^{\mathrm{a}}$, Ana M. Benito ${ }^{\mathrm{a} *}$

an Instituto de Carboquímica (ICB-CSIC). C/ Miguel Luesma Castán,4. E-50018 Zaragoza (Spain

${ }^{b}$ Laboratory of Separation and Reaction Engineering - Laboratory of Catalysis and Materials (LSRELCM), Faculdade de Engenharia, Universidade do Porto, Rua Dr. Roberto Frias, 4200-465 Porto, Portugal.

\section{Abstract}

Graphene oxide (GO) and related materials are widely reported to enhance the photocatalytic activity of zinc oxide. However, the origin of the observed performance improvements remains elusive and studies contributing to a deeper understanding of this critical issue are largely missing. In this work, we have prepared a set of benchmark ZnO-GO hybrid materials in order to systematically put under closer scrutiny the influence of the surface chemistry of GO on the photocatalytic degradation of methylene blue. The set of $\mathrm{ZnO}-\mathrm{GO}$ hybrids has been synthesized in an ultrasonication process involving $\mathrm{ZnO}$ nanoparticles obtained in a microwave synthesis process and $\mathrm{GO}$ with three distinct oxidation degrees, employed in three different loading fractions. Structural and physical-chemical characterization by XRD, FTIR, Raman, UV-Vis, photoluminescence and spectroscopy and XPS, consistently demonstrate the importance of the surface chemistry of GO for establishing photo-induced charge-transfer interface interactions with $\mathrm{ZnO}$, facilitating the enhancement of the catalytic activity of the $\mathrm{ZnO}$ -

\footnotetext{
* Corresponding author E-mail address: abenito@icb.csic.es (A.M. Benito)
} 
$\mathrm{GO}$ catalyst. Optimized interface interactions thus enabled the design of a $\mathrm{ZnO}-\mathrm{GO}$ catalyst exhibiting a conversion rate of $80 \%$ obtained in a time of 70 minutes and at a catalyst concentration of only $0.045 \mathrm{mg} / \mathrm{mL}$.

\section{Keywords}

graphene oxide; zinc oxide; $\mathrm{ZnO}-\mathrm{GO}$ hybrid; photocatalysis; surface chemistry

\section{Introduction}

Graphene oxide (GO) has become the object of many research studies in the last years for its multifunctional character based on its large surface area, amphiphilicity, and tunable electronic properties by surface modification $[1,2]$. GO is obtained by oxidation and exfoliation of graphite $[3,4]$ and consists of a single graphenic sheet with $\pi$-conjugated $\mathrm{sp}^{2}$ domains together with regions of $\mathrm{sp}^{3}$ character, which contain oxygen functional groups (oFGs) covalently attached to the carbon atoms, both in the basal plane and on the edges. It can be described as a wide band-gap semiconducting material with a highly complex non-stoichiometric chemical structure [5-7]. Exhibiting additionally a much higher hydrophilic character than graphene, further facilitates its processing in aqueous media and, therefore, its use in a wide variety of technological applications $[8,9]$. Moreover, the chemically active surface of GO enables the attachment of different organic molecules or metal oxides that modify its electronic properties. This surface-tuning flexibility is of particular interest in photocatalysis, since GO can form hybrids in combination with photocatalytically active semiconducting metal oxides, improving their photocatalytic response $[10,11]$. One of the most studied metal oxides has been zinc oxide $(\mathrm{ZnO})$ since it exhibits several advantages including low production cost, chemical stability, non-toxicity, high photosensitivity and wide band gap (3.37 eV) [12-14]. Its nanostructuration, moreover, opens new possibilities in 
photocatalysis as it enables higher efficiency and facilitates the ulterior recovery of the photocatalyst $[15,16]$. Additionally, its combination with GO results in remarkable photocatalytic performance [16-20] since GO has an active role in photocatalysis by decreasing the aggregation of $\mathrm{ZnO}$ particles and acting as electron-acceptor material, hindering the electron-hole recombination [17, 21-23]. Furthermore, graphene has been proven to enhance adsorption of organic pollutants on the hybrid surface probably due to the $\pi-\pi$ interaction between the aromatic pollutants and the $\mathrm{sp}^{2}$ region of graphene, which eventually improves the photocatalytic efficiency [24]. Also, GO induces the formation of oxygen vacancies in $\mathrm{ZnO}$ improving its photoresponse [25]. Likewise, increased loading fractions of GO are reported to significantly enhance the photocatalytic performance up to certain threshold values. [22].

A vast majority of literature studies claims that GO should be transformed to rGO in ZnO-GO composites to achieve photocatalytic enhancement effects. Hereto processes either involving the direct mixing of (functionalized) $\mathrm{rGO}$ with $\mathrm{ZnO}$ or thermal postreduction of a $\mathrm{ZnO}-\mathrm{GO}$ composites are reported [26-30]. Consequently, the superior conducting properties of rGO, combined with its high surface area are made responsible for the improvement of the photocatalytic activity. While this usually is explained in a rather general way, the deeper reasons are not fully understood.

On the other hand, our former studies revealed that even non-reduced GO is able to improve the photocatalytic activity in $\mathrm{TiO}_{2}-\mathrm{GO}$ composites $[31,32]$. Here it was shown that the enhancement effect strongly depends on the applied synthesis method and the fact to achieve a perfect coverage of $\mathrm{TiO}_{2}$ nanoparticles by GO. It was demonstrated that the oxygen functional groups mediate the efficient and uniform assembly of the $\mathrm{TiO}_{2}$ nanoparticles on the GO sheets, leading to highly efficient photocatalysts both under near-UV/Vis and visible light, when an optimum amount of GO was employed. 
On the contrary, using synthesis conditions not resulting in the formation of $\mathrm{TiO}_{2}$ nanoparticles perfectly covered by GO, i.e. comprised of $\mathrm{TiO}_{2}$ nanoparticles not well connected to GO sheets, improvement of the photocatalytic properties only can be obtained through the use of rGO.

These findings clearly underline that the conductivity of GO, i.e. the presence of rGO, does not constitute a fundamental intrinsic requirement for obtaining improved photocatalytic activity and that certain critical issues, not looked-up yet, need to be scrutinized in more detail

In this article, we focus on the impact of the oxygen functional groups of GO for achieving enhanced photocatalytic activity in $\mathrm{ZnO}-\mathrm{GO}$ hybrid materials. By using GO with three distinct degrees of oxygen groups we systematically study the influence of the surface chemistry of GO on the photocatalytic activity of $\mathrm{ZnO}-\mathrm{GO}$ hybrids for the degradation of methylene blue under UV-light irradiation. Thorough analyses by various physicochemical techniques provide evidence on the establishment of specific interface interactions between $\mathrm{ZnO}$ and $\mathrm{GO}$, and how this influences the final photocatalytic behavior. Moreover, we demonstrate that by solely controlling the oxidation degree of GO and by optimizing the loading fraction highly efficient $\mathrm{ZnO}-\mathrm{GO}$ catalysts can be prepared, without any further need to apply tedious reduction steps. The performance is tested in photocatalytic degradation reactions of methylene blue and diphenhydramine (up-scaling and recyclability), as well as in the photoelectrocatalytic oxidation of water.

\section{Experimental}

\subsection{Materials}


Graphite flakes, zinc acetate dihydrate $\left(\mathrm{ZnAc} \cdot 2 \mathrm{H}_{2} \mathrm{O}\right)$, Titanium (IV) oxide $\left(\mathrm{TiO}_{2} \mathrm{P} 25\right)$, sodium nitrate $\left(\mathrm{NaNO}_{3}\right)$, sulfuric acid $\left(\mathrm{H}_{2} \mathrm{SO}_{4}\right)$, hydrochloric acid $(\mathrm{HCl})$, potassium permanganate $\left(\mathrm{KMnO}_{4}\right)$, hydrogen peroxide $\left(\mathrm{H}_{2} \mathrm{O}_{2}\right)$, potassium chloride $(\mathrm{KCl})$, sodium sulfate (anhydrous), methanol, methylene blue (MB) and diphenhydramine (DP) were purchased from Aldrich and used as received.

\subsection{Synthesis of $\mathrm{ZnO}$ nanoparticles}

$\mathrm{ZnO}$ nanoparticles were synthesized by a sol-gel procedure based on microwave irradiation of a solution of zinc acetate dehydrated ( $\mathrm{ZnAc})$ in ethylene glycol (EG), using a CEMTM S-class Microwave Synthesis System. Briefly, $0.025 \mathrm{~mol}$ of ZnAc solved in $0.5 \mathrm{ml}$ of deionized water were added to $250 \mathrm{ml} \mathrm{EG}$, and the obtained solution was microwave heated under stirring at $160^{\circ} \mathrm{C}$ for $5 \mathrm{~min}$, using $300 \mathrm{~W}$ of power. The $\mathrm{ZnO}$ precipitate was collected by centrifugation, washed repeatedly with deionized water and dried in an oven at $100{ }^{\circ} \mathrm{C}$.

\subsection{Synthesis of graphene oxide with different oxidation degrees (GOX)}

First, graphite oxide was prepared using a modified Hummers method as reported elsewhere $[3,33]$. Specifically, $5 \mathrm{~g}$ of graphite flakes were put into a mixture of $170 \mathrm{ml}$ $\mathrm{H}_{2} \mathrm{SO}_{4}$ and $3.75 \mathrm{~g} \mathrm{NaNO}_{3}$, and the mixture was cooled in an ice bath and stirred for 30 minutes. Thereafter, $25 \mathrm{~g}$ of $\mathrm{KMnO}_{4}$ were slowly added and stirred for another 30 minutes. Then, the ice bath was removed and the mixture warmed to $35-40{ }^{\circ} \mathrm{C}$ and stirred for different times ( 2 h, $4 \mathrm{~h}$ and overnight), yielding graphite oxide with increasing oxidation degree. The reaction was terminated by slowly adding $250 \mathrm{ml}$ of deionized water and then $20 \mathrm{ml} \mathrm{H}_{2} \mathrm{O}_{2}(30 \%)$ solution. The resulting dispersion was filtered and the obtained powder material was repeatedly washed with $400 \mathrm{ml}$ of $\mathrm{HCl}$ : $\mathrm{H}_{2} \mathrm{O}(1: 10 \mathrm{v} / \mathrm{v})$ to remove metal ions followed by deionized water until neutral $\mathrm{pH}$. Finally, the graphite oxide obtained was dried at room temperature. 
Graphene oxide was obtained by bath sonication $(45 \mathrm{kHz})$ of an aqueous graphite oxide dispersion $(2 \mathrm{mg} / \mathrm{ml})$ for 2 hours, followed by centrifugation at $4500 \mathrm{rpm}$ during 60 minutes, obtaining a brown-colored dispersion of exfoliated GOX $(X=1,2$ or 3 , for the oxidation during $2 \mathrm{~h}, 4 \mathrm{~h}$, and overnight, labeled as the lowest, medium and highest oxidation degree, respectively) with a final concentration of $1 \mathrm{mg} / \mathrm{ml}$.

\subsection{Preparation of $\mathrm{ZnO}-G O X$ catalysts}

Catalysts were prepared in solution by a novel method based on ultrasonic mixing and subsequent freeze-drying. $\mathrm{ZnO}$ powder was added to a $0.5 \mathrm{mg} / \mathrm{ml}$ aqueous dispersion of graphene oxide (GO1, GO2 or GO3) in the convenient amount to obtain the required $\mathrm{ZnO}$ to GOX ratio $(3: 1,10: 1$ or $20: 1 \mathrm{w} / \mathrm{w})$. The resulting dispersion was bath sonicated for 20 minutes, frozen and freeze-dried for 24 hours to obtain the $\mathrm{ZnO}-\mathrm{GOX}$ catalysts in the form of a fine powder.

\subsection{Characterization}

The surface morphology of the synthesized materials was investigated by scanning electronic microscopy (SEM) using a Hitachi S-3400N microscope and transmission electron microscopy (TEM) employing a JEOL-2000 FXII. Elemental analysis of the GOX materials was performed in a Thermo Flash 1112 analyzer. The sample in Ag capsules was pyrolyzed at $1070{ }^{\circ} \mathrm{C}$ under He flow. The produced gases flow through a reducing bed of carbon black that transforms all $\mathrm{CO}_{\mathrm{x}}$ into $\mathrm{CO}$. Subsequently, the gases flow through a polar chromatographic column that separates $\mathrm{CH}_{4}$ from $\mathrm{CO}$ and the latter is detected and quantified using a thermal conductivity detector calibrated with sulphanilamide.

Powder X-ray diffraction (XRD) patterns were obtained using a Bruker AXS D8 Advance diffractometer provided with a $\mathrm{Cu} K \alpha$ as X-ray irradiation source $\left(\lambda_{\mathrm{Cu} \mathrm{K} \alpha}=\right.$ 
$1.541 \AA$ ) through the $2 \theta$ range from $20^{\circ}$ to $80^{\circ}$. The software EVA and the Powder Diffraction File (PDF) database were utilized for the semi-quantitative estimate of the phase composition. The size of crystal domains was calculated by the Scherrer equation with a shape factor of 0.89 . Crystallite sizes were not calculated for phase contents of less than $5 \%$, as the error was very high. Fourier-transform infrared (FTIR) spectra in $\mathrm{KBr}$ powder-pressed pellets were recorded in a Bruker Vertex 70 spectrometer. Raman spectroscopy was performed using a Horiba Jobin Yvon spectrometer (model HR 800 UV) working with a green laser (excitation $\lambda=532 \mathrm{~nm}$ ). X-ray photoelectron spectroscopy (XPS) of the powder materials was performed in an ESCAPlus Omicron spectrometer provided with an $\mathrm{Mg}$ anode $(1253.6 \mathrm{eV})$ working at $225 \mathrm{~W}(15 \mathrm{~mA}, 15$ $\mathrm{kV})$ and the peaks were calibrated with C1s peak $(284.5 \mathrm{eV})$. The XPS peaks were fitted to a GL(70) Voigt lineshape (a combination of 70\% Gaussian and $30 \%$ Lorentzian character), performing a Shirley background subtraction. Asymmetry for the $\mathrm{sp}^{2}$ peak of the $\mathrm{C} 1 \mathrm{~s}$ spectra was defined as a function $\operatorname{LA}(\alpha, \beta, \mathrm{m})$, where $\alpha=0.4$ and $\beta=0.38$ describe the spread of the tail on each side of the Lorentzian component and the parameter $m=70$ specifies the width of the Gaussian used to convolute the Lorentzian curve.

The optical properties of the materials were characterized by a UV-Vis spectrometer (Shimadzu UV-3600) from 190 to $900 \mathrm{~nm}$. Photoluminescence emission spectra (PL) were recorded at $350 \mathrm{~nm}$ of excitation in a Horiba Jobin Yvon Fluoromax-P, slits of excitation and emission at $1 \mathrm{~mm}$. All the spectra were recorded at room temperature using $10 \mathrm{~mm}$ path-length quartz cuvette.

\subsection{Photocatalytic Experiments}


The photocatalytic activity of the synthesized materials was evaluated based on the degradation of methylene blue (MB) in aqueous solution under UV light irradiation. Photocatalytic activity of a commercial $\mathrm{TiO}_{2}$ photocatalyst (P25) was also measured and used as reference. An UV lamp (Philips TL8W (Hg)) was utilized as a radiation source. The lamp emitted a minute fraction of the total radiation at 324 and $325 \mathrm{~nm}$ and the rest between 342 and $400 \mathrm{~nm}$ with a maximum irradiance peak at $365 \mathrm{~nm}(3.4 \mathrm{eV})$. In a typical experiment, $3.3 \mathrm{ml}$ of $\mathrm{MB}$ aqueous solution $(0.015 \mathrm{mg} / \mathrm{ml})$ and the corresponding photocatalyst $(0.045 \mathrm{mg} / \mathrm{ml})$ were mixed and magnetically stirred in a quartz cuvette during $30 \mathrm{~min}$ in dark conditions before switching on the lamp, in order to achieve the adsorption-desorption equilibrium condition. The cuvette, placed $5 \mathrm{~cm}$ away from light source, was irradiated under UV light without a filter. Degradation of methylene blue was analyzed by recording the absorbance of the solutions at the maximum absorption band $(\lambda=663.5 \mathrm{~nm})$ of $\mathrm{MB}$, using a $\mathrm{UV}$-vis spectrometer at given time intervals.

The degradation efficiency of the photocatalyst was calculated using the following equation:

Degradation $(\%)=\left(\mathrm{A}_{0}-\mathrm{A}_{t} / \mathrm{A}_{0}\right) \times 100 \%$

Where $A_{0}$ is the absorbance values of MB at initial stage and $A_{t}$ is the absorbance of MB at the different time intervals measured. The photodegradation of MB follows pseudo-first-order kinetics, which can be expressed as follows:

$\operatorname{Ln}\left(A_{t} / A_{0}\right)=-k t$

Where $\mathrm{k}\left(\mathrm{min}^{-1}\right)$ is the degradation rate constant [34].

\subsection{Photoelectrochemical measurements.}


Fluorinated tin oxide (FTO) substrates of $2.5 \times 1 \mathrm{~cm}$ (Solems SA, France) were sonicated in isopropanol for 5 minutes before spraying. $\mathrm{ZnO}$ films were prepared by spraying a $\mathrm{ZnO}$ (or $\mathrm{ZnO}-\mathrm{GO})$ suspension in ethanol $(0.5 \mathrm{mg} / \mathrm{ml})$ onto the FTO substrates using an automatized spray coater (Nadetech ND-SP, Spain), covering an area of $0.7 \mathrm{~cm}^{2}$. All the photoelectrochemical measurements were performed using $\mathrm{N}_{2}$-purged $0.1 \mathrm{M} \mathrm{Na}_{2} \mathrm{SO}_{4}$ as supporting electrolyte, a $\mathrm{Ag} / \mathrm{AgCl}(3 \mathrm{M} \mathrm{NaCl})$ as reference electrode and a graphite rod as counter electrode, the scan rate was $20 \mathrm{mV} / \mathrm{s}$ for all cyclic voltammetry (CV) experiments. The $\mathrm{ZnO}-\mathrm{GO}$ electrodes were electrochemically reduced by cycling from 0.4 to $-1.0 \mathrm{~V}$ until a constant voltammogram is attained (eight cycles) in order to obtain the electrochemically reduced $\mathrm{ZnO}-\mathrm{ECrGO}$ electrodes. Photentiostatic chronoamperograms were recorded at $0.4 \mathrm{~V}$.

\subsection{Proof of concept: up-scaling and recyclability of ZnO-GO hybrid catalysts}

The photodegradation of diphenhydramine (DP) was carried out at room temperature $\left(25^{\circ} \mathrm{C}\right)$ in a homemade batch reactor, using $7.5 \mathrm{~mL}$ of reaction medium in each experiment. A magnetic stirrer was placed inside to homogenize the sample and the reactor was housed within a cooling system, all of it located at $5 \mathrm{~cm}$ of the UV light source $\left(\sim 50 \mathrm{~mW} / \mathrm{cm}^{2}\right)$. A flow of oxygen was continuously supplied to the reactor, and aliquots were sampled at specific times. The catalyst was separated by filtration at each sampled aliquot. The concentration of DP was fixed in all experiments at $100 \mathrm{mg} / \mathrm{L}$, and the concentration of catalyst was $1 \mathrm{~g} / \mathrm{L}$. The reaction mixture (with the suspended hybrid catalyst) was kept in the dark under stirring (30 min), turning on the light source afterwards, and sampling $1 \mathrm{~mL}$ aliquots at each desired time. The detection of DP was carried out with an HPLC equipment (Hitachi Elite La Chromsystem), provided with a Hydrosphere C18 column ( $250 \mathrm{~mm} \times 4,6 \mathrm{~mm}, 5 \mu \mathrm{m}$ particle size). The analyses are based on an isocratic method, with a $1 \mathrm{~mL} / \mathrm{min}$ flow rate of the eluent $(\mathrm{A}: \mathrm{B}, 30: 70 \mathrm{v} / \mathrm{v}$ 
of $20 \mathrm{nM} \mathrm{NaH}_{2} \mathrm{PO}_{4}$ acidified with $\mathrm{H}_{3} \mathrm{PO}_{4}$ at $\mathrm{pH}=2.80$ (A), and acetonitrile (B)), providing a linear response over the whole experimental range.

\section{Results and discussion}

\subsection{Influence of the $G O$ oxidation degree on the catalytic activity of the $\mathrm{ZnO}-G O X$} catalysts.

Graphene oxide with three different oxidation degrees (GOX) was prepared from their graphite oxide counterparts by modifying the oxidation time from two hours (GO1) to four hours (GO2) and overnight (GO3). Elemental analysis (Table 1) of the GOX materials reveals a progressive increase of the oxygen content from $39 \mathrm{wt} . \%$ in GO1 to 46 wt.\% in GO3, confirming the different oxidation degree of the obtained GOX materials. Next, ZnO-GOX hybrid materials based on GO1, GO2 or GO3, and ZnO with 3:1 w/w of ZnO:GOX ratio were prepared and characterized.

Table 1. Elemental Analysis of the different graphene oxide materials. $N$ and $S$ impurities come from the harsh oxidation procedure applied to graphite. Please, note that elemental analysis results do not consider the physisorbed water contribution.

\begin{tabular}{cccccc}
\hline $\begin{array}{c}\text { Graphene } \\
\text { Oxide }\end{array}$ & O (wt.\%) & C (wt.\%) & H (wt.\%) & N (wt.\%) & S (wt.\%) \\
\hline GO1 & 38.8 & 47.2 & 2.8 & 0.1 & 1.1 \\
GO2 & 42.6 & 41.5 & 3.3 & 0.0 & 1.0 \\
GO3 & 46.0 & 40.8 & 3.1 & 0.0 & 1.4 \\
\hline
\end{tabular}

Electronic microscopy analysis (Fig. S1) confirms the deposition of $\mathrm{ZnO}$ nanoparticles ranging from 10 to $30 \mathrm{~nm}$ on the surface of graphene oxide. However, no strong morphological differences are observed for the different types of hybrids.

Fig. 1 shows the XRD patterns of the $\mathrm{ZnO}$ nanoparticles and the three $\mathrm{ZnO}-\mathrm{GOX}$ hybrids with equal GOX content (ZnO:GOX ratio equal to 3:1). All the diffraction peaks of the pure $\mathrm{ZnO}$ material can be well indexed to the hexagonal wurtzite phase of $\mathrm{ZnO}$, confirming that only the nanocrystalline $\mathrm{ZnO}$ phase was detected. No other 
crystalline phases were observed. The sharp diffraction peaks evidence the good crystallinity of the prepared particles. The crystalline lattice parameters for the $\mathrm{ZnO}$ were $\mathrm{a}=3.251 \AA$ and $\mathrm{c}=5.208 \AA$, being $\mathrm{c} / \mathrm{a}=1.601$, close to an ideal hexagonal structure $(\mathrm{c} / \mathrm{a}=1.633)$ [35]. The crystallite size of the $\mathrm{ZnO}$ nanoparticles calculated by the X-ray line broadening method using the Debye-Scherrer's equation is $10 \mathrm{~nm}$.

XRD patterns of the ZnO-GOX materials (Fig. 1) are similar to the XRD pattern of the pristine $\mathrm{ZnO}$, confirming that the wurtzite structure of the $\mathrm{ZnO}$ nanoparticles is maintained. However, no evidence on the presence of GOX diffraction peaks (Fig. S2) is found in the XRD diffractograms, indicating that the GOX materials are well exfoliated, and their contribution is masked by the strong scatter of $\mathrm{ZnO}$, which has much more electron density. Also, no shift was observed in the position of $\mathrm{ZnO}$ diffraction peaks compared to pristine $\mathrm{ZnO}$, evidencing that the native crystalline structure of $\mathrm{ZnO}$ is maintained after preparation of the hybrid material. Interestingly, the relative intensity ratios of the three main diffraction peaks, (100), (002) and (101) vary when GOX with different oxidation degree is used, suggesting the establishment of interfacial interactions between $\mathrm{ZnO}$ and GOX. To see this more clearly, Table 2 shows the calculated $\mathrm{I}_{\mathrm{i}} / \mathrm{I}_{0}$ relative intensity ratios for the three main diffraction peaks, i.e. the intensity of a particular diffraction peak $\left(\mathrm{I}_{\mathrm{i}}\right)$ relative to the intensity of the most intense diffraction peak $\left(\mathrm{I}_{0}\right)$, the (101). A significant increase of the (002) relative intensities, especially in the case of the $\mathrm{ZnO}-\mathrm{GO} 3$, supports a preferential interaction through the (002) plane of the $\mathrm{ZnO}$. The crystalline lattice parameters of the $\mathrm{ZnO}-\mathrm{GOX}$ materials were similar to the $\mathrm{ZnO}$ parameters $(\mathrm{a}=3.251 \AA$ and $\mathrm{c}=5.208 \AA)$ and only the $\mathrm{ZnO}$ GO3 has a slightly different c/a ratio (1.603). The calculated crystallite size of $\mathrm{ZnO}$ within the hybrids was 16.6, 22.64 and $21 \mathrm{~nm}$ for the $\mathrm{ZnO}-\mathrm{GO} 1, \mathrm{ZnO}-\mathrm{GO} 2$ and $\mathrm{ZnO}-$ 
GO3, respectively. Thus, the values for $\mathrm{ZnO}-\mathrm{GOX}$ hybrids are significantly larger than the ones calculated for the bare $\mathrm{ZnO}$ nanoparticles.

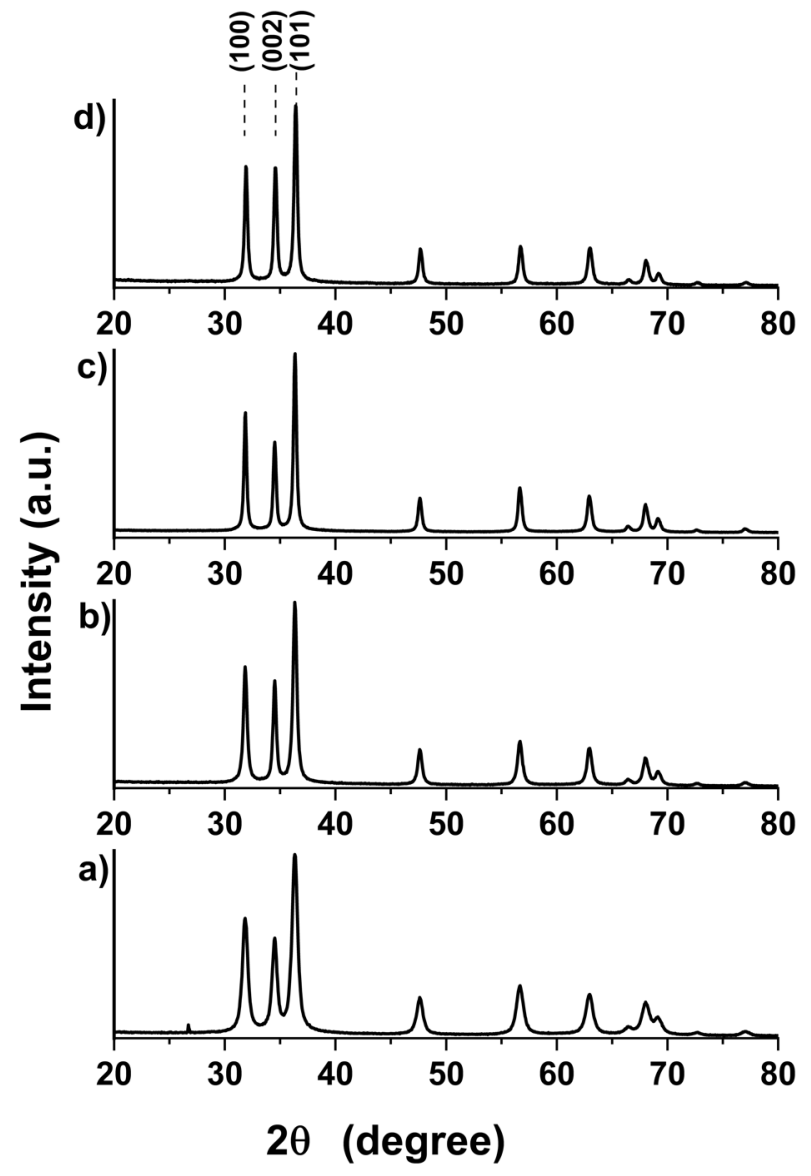

Fig. 1. XRD pattern of a) $\mathrm{ZnO}$, and b) $\mathrm{ZnO}-\mathrm{GO} 1(1: 3 \mathrm{w} / \mathrm{w})$, c) $\mathrm{ZnO}$ GO2 (1:3 w/w), d) $\mathrm{ZnO}-\mathrm{GO} 3(1: 3 \mathrm{w} / \mathrm{w})$

Table 2. Relative intensity $\left(\mathrm{I}_{\mathrm{i}} / \mathrm{I}_{0}\right)$ of the three main diffraction peaks (100), (002) and (101) with respect to the most intense (101) for the $\mathrm{ZnO}$ and $\mathrm{ZnO}-\mathrm{GO}(3: 1 \mathrm{w} / \mathrm{w})$ materials.

\begin{tabular}{cccccc}
\hline \multirow{2}{*}{$\begin{array}{c}\text { Miller index } \\
(\mathbf{h}, \mathbf{k}, \mathbf{l})\end{array}$} & \multirow{20}{*}{$\begin{array}{c}\text { I } \\
\text { (degree) }\end{array}$} & ZnO & ZnO-GO1 & ZnO-GO2 & ZnO-GO3 \\
\cline { 3 - 6 }$(\mathbf{1 0 0 )}$ & 31.8 & 0.65 & 0.65 & 0.67 & 0.67 \\
$\mathbf{( 0 0 2 )}$ & 34.5 & 0.55 & 0.58 & 0.51 & 0.66 \\
$(\mathbf{1 0 1})$ & 36.3 & 1 & 1 & 1 & 1 \\
\hline
\end{tabular}

Raman spectroscopy evidences the presence of graphene oxide in the hybrids. The Raman spectra of the GOX materials (Fig. 2) show the characteristic D $\left(1358 \mathrm{~cm}^{-1}\right)$ and 
$\mathrm{G}\left(1592 \mathrm{~cm}^{-1}\right)$ bands of the graphenic materials. The D band is attributed to vibrations of the carbons with $\mathrm{sp}^{3}$ character and, hence, indicative of structural imperfections, possibly due to the attachment of oFGs on the carbon basal plane and other induced defects during harsh oxidation. The $\mathrm{G}$ band is extremely sensitive to $\mathrm{sp}^{2}$ carbon in-plane vibrations. The spectra of $\mathrm{ZnO}-\mathrm{GOX}$ show a shift for both $\mathrm{D}$ and $\mathrm{G}$ bands located at 1347 and $1598 \mathrm{~cm}^{-1}$, respectively. This shift suggests an interaction between $\mathrm{ZnO}$ and GO. Importantly, an additional Raman mode emerges at around $1100 \mathrm{~cm}^{-1}$ in the spectra of the $\mathrm{ZnO}-\mathrm{GOX}$ hybrids. This mode, not visible in pure $\mathrm{ZnO}$ (Fig. S4), seems to belong to GOX since a very low intensity shoulder is here observable (Fig. 2). However, it is in the Raman of the $\mathrm{Zn}-\mathrm{GO}$ hybrids where the signal at $1100 \mathrm{~cm}^{-1}$ is more evident, especially when less oxidized GOX (GO1 or GO2) is used. The increase of the peak intensity also suggests that an interfacial interaction between $\mathrm{ZnO}$ and $\mathrm{GO}$ is established. The intensity of the D band with respect to the $\mathrm{G}$ band is typically used to characterize the level of disorder in graphene materials [36]. The calculated $\mathrm{I}_{\mathrm{D}} / \mathrm{I}_{\mathrm{G}}$ ratio is $0.65,0.67$ and 0.62 for $\mathrm{GO} 1, \mathrm{GO} 2$ and $\mathrm{GO} 3$, respectively. And the $\mathrm{I}_{\mathrm{D}} / \mathrm{I}_{\mathrm{G}}$ ratio increases in the hybrids, being $0.95,0.81$, and 0.80 for Zn-GO1, Zn-GO2, and Zn-GO3, respectively. This higher $I_{D} / I_{G}$ ratio evidences a higher level of disorder, most likely denoting an advantageous integration of $\mathrm{GO}$ with $\mathrm{ZnO}$ [36]. 

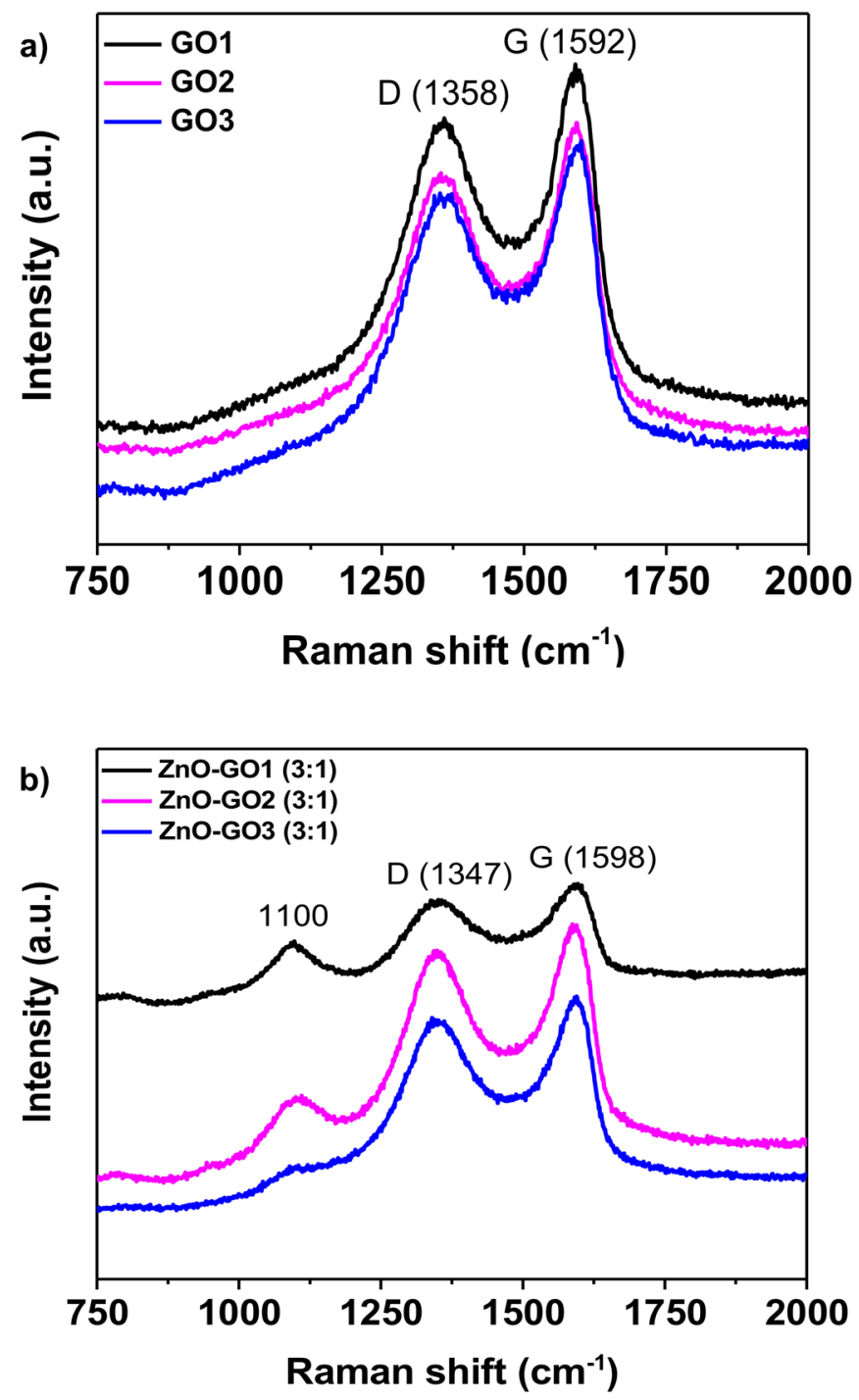

Fig 2. Raman spectra of GOX and $\mathrm{ZnO}-\mathrm{GOX}(3: 1 \mathrm{w} / \mathrm{w})$ materials

FTIR spectra of the GOX materials (Fig. 3, Fig. S5) confirm their high hydrophilic character, as they show an intense broad band in the $3000-3600 \mathrm{~cm}^{-1}$ region due to the stretching vibrations of $\mathrm{O}-\mathrm{H}$ groups coming from alcohols, carboxylic acids and water. This band increases with the oxidation degree of GOX. Water scissor mode (deformation vibration) is evidenced in the $1600-1700 \mathrm{~cm}^{-1}$ region, where it overlaps with the asymmetric stretch of $\mathrm{sp}^{2} \mathrm{C}=\mathrm{C}$ vibration modes that should appear at 1500 $1600 \mathrm{~cm}^{-1}$ and are hidden by the strong water contribution [6]. $\mathrm{C}=\mathrm{O}$ stretching mode band is observed at $1734 \mathrm{~cm}^{-1}$, compatible with ester/lactone groups. The characteristic 
C-O peaks appear in the $900-1500 \mathrm{~cm}^{-1}$ region where the presence of overlapped modes from several distinct species impedes an unequivocal assignation. Here signals at 1223 and $1060 \mathrm{~cm}^{-1}$ are assigned to alcohol $(\mathrm{C}-\mathrm{OH})$ and epoxy (C-O-C) groups, respectively. Increasing the oxidation degree of GOX leads to the increase of the $\mathrm{OH}$ band at 3415 $\mathrm{cm}^{-1}$ and to a more intense water scissor mode at $1631 \mathrm{~cm}^{-1}$ together with the increase of the $\mathrm{C}-\mathrm{O}$ bands. No modification of the $\mathrm{C}=\mathrm{O}$ signal is observed, which has interesting implications in the resulting $\mathrm{ZnO}-\mathrm{GOX}$ hybrids as will show below.

FTIR spectra of the ZnO-GOX materials show the main features of GOX spectra with the clear emergence of the intense band at $457 \mathrm{~cm}^{-1}$ characteristic of the stretching vibrations of $\mathrm{Zn}-\mathrm{O}$ [37]. The most significant difference with respect to the GOX is the disappearance of the $\mathrm{C}=\mathrm{O}$ band at $1734 \mathrm{~cm}^{-1}$, evidencing an interaction between $\mathrm{ZnO}$ and GOX through the $\mathrm{C}=\mathrm{O}$ groups of GOX.

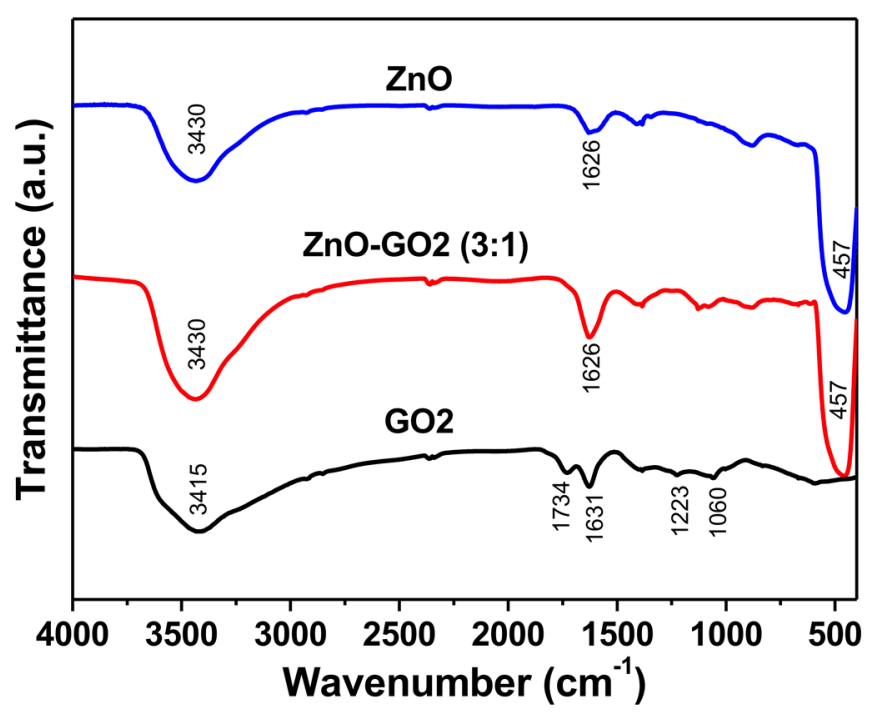

Fig 3. FTIR spectra of GO2, $\mathrm{ZnO}$ and $\mathrm{ZnO}-\mathrm{GO} 2(3: 1 \mathrm{w} / \mathrm{w})$.

UV-Vis absorption spectra of the GOX materials (Fig. 4) show the characteristic absorption peak at $230 \mathrm{~nm}$ due to the $\pi-\pi^{*}$ transition of $\mathrm{C}=\mathrm{C}$ bonds and a abroad shoulder at around $310 \mathrm{~nm}$, corresponding to $\mathrm{n}-\pi^{*}$ transition of $\mathrm{C}=\mathrm{O}[8]$. $\mathrm{ZnO}$ nanoparticles exhibit 
the typical absorption edge at $374 \mathrm{~nm}$, indicating the existence of highly crystalline $\mathrm{ZnO}$ nanostructure. The $\pi-\pi^{*}$ transition characteristic of GOX is hardly observable in the UVvis of the $\mathrm{ZnO}-\mathrm{GOX}$ composites (Fig. 4). Interestingly, an absorption at $250 \mathrm{~nm}$ is now visible, suggesting a partial reduction of the GOX in the hybrid [8]. This is accompanied by a blue-shift of the $\mathrm{ZnO}$ absorption edge to $366 \mathrm{~nm}$ evidencing an interaction with GO. Corresponding shifts are indicated in Fig. 4b, c by dashed lines.

The photoluminescence spectra of $\mathrm{ZnO}$ and $\mathrm{ZnO}-\mathrm{GO}$ materials are shown in Fig 5. A quenching of the emission of $\mathrm{ZnO}$ photoluminescence is clearly observed for the hybrid materials. Moreover, when GO is added, the strong emission at $382 \mathrm{~nm}$ of the $\mathrm{ZnO}$ blueshifts to $375 \mathrm{~nm}$ and decreases in intensity with respect to the other emission features. The quenching is ascribed to the electron transfer from the conduction band of $\mathrm{ZnO}$ (good electron donor) to the valence band of GO (electron acceptor). This charge transfer, sensitive to suitable interactions at the interface between $\mathrm{ZnO}$ and $\mathrm{GO}$, thus reduces the recombination of photogenerated electron-hole pairs. Consequently, the availability of additional charge carriers in $\mathrm{ZnO}-\mathrm{GO}$ hybrids is expected to enhance photocatalytic performance of $\mathrm{ZnO}$. 

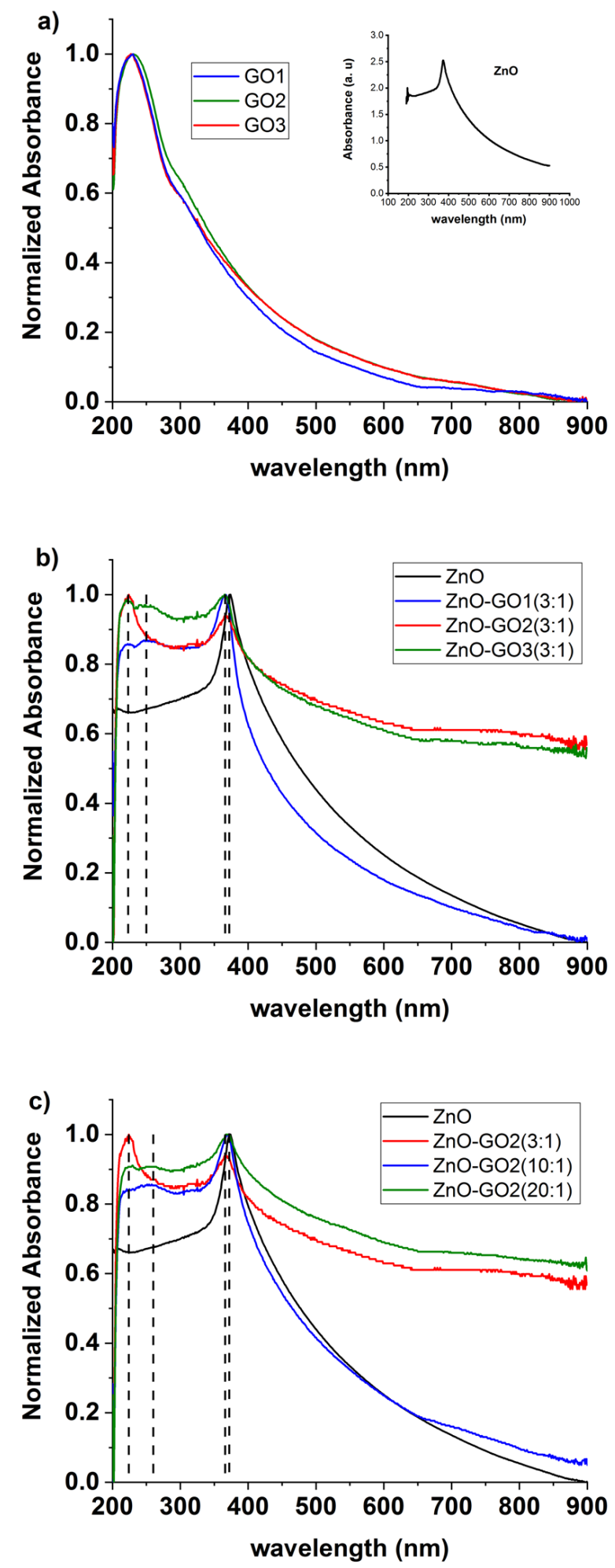

Fig 4. UV-vis spectra of (a) pristine GOX and $\mathrm{ZnO}$ materials, (b) $\mathrm{ZnO}, \mathrm{ZnO}$ GOX (3:1 w/w) hybrids; (c) $\mathrm{ZnO}$ and $\mathrm{ZnO}-G O 2$ hybrids. 

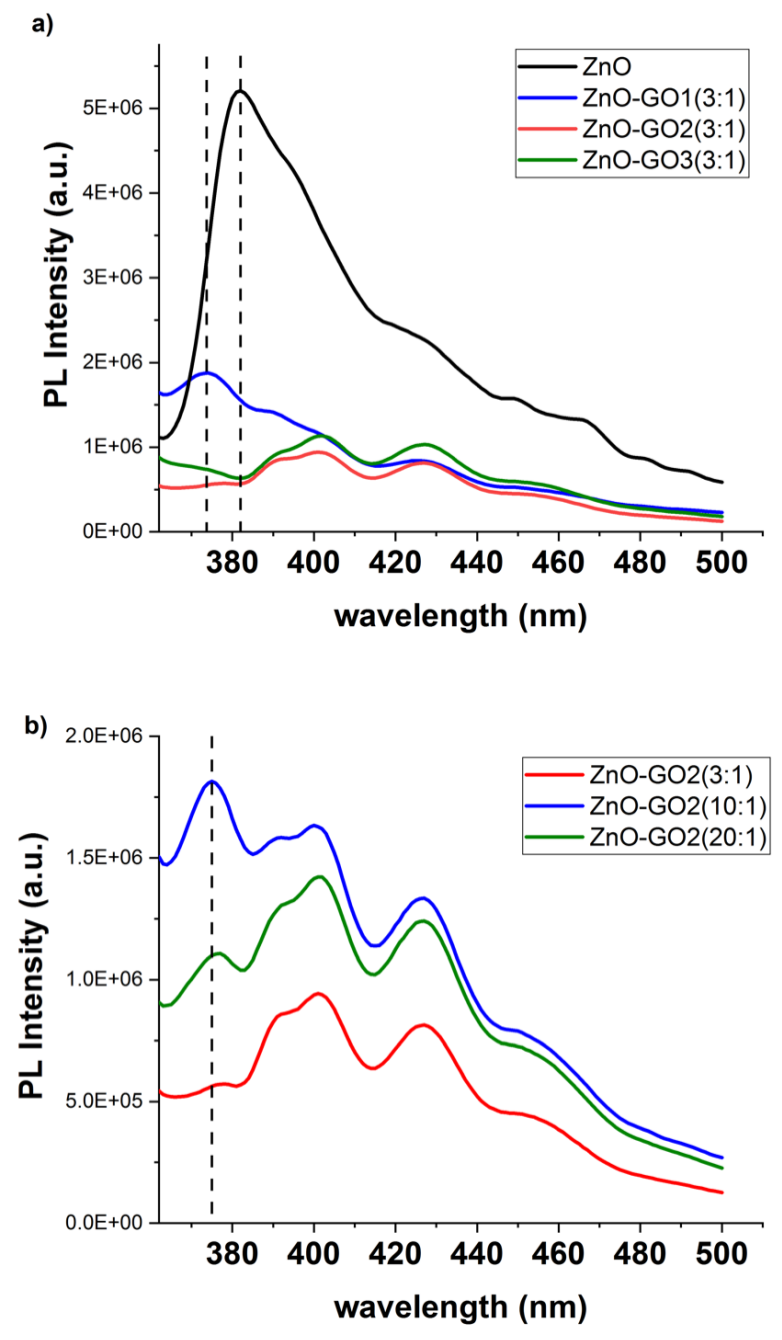

Fig 5. Photoluminescence spectra of the pristine $\mathrm{ZnO}$ and $\mathrm{ZnO}-$-GO hybrid materials

Next, the photocatalytic activity of the synthesized catalysts ( $\mathrm{ZnO}$ and the $\mathrm{ZnO}-\mathrm{GOX}$ ) was analyzed by measuring the intensity of the UV-Vis absorption of the maximum absorption band of MB (663.5 nm) during its degradation. During the equilibration period, prior to the photocatalysis experiments, no adsorption of MB in the catalyst was observed due to the small amount of catalyst used and due to the very low surface area of the prepared catalytic materials $\left(6 \mathrm{~m}^{2} / \mathrm{g}\right.$ for $\mathrm{ZnO}$ and 30 to $38 \mathrm{~m}^{2} / \mathrm{g}$ for the hybrid materials). Photolysis experiments performed under UV light (without catalyst) and 
experiments under dark conditions (with catalyst) did not show any apparent degradation of MB. The use of GOX under light as catalyst did not show any evidence for MB degradation either. ZnO-GOX hybrids show significant enhancement in the photodegradation of $\mathrm{MB}$ compared to bare $\mathrm{ZnO}$, being $\mathrm{ZnO}-\mathrm{GO} 1$ and $\mathrm{ZnO}-\mathrm{GO} 2$ the most effective catalysts (Fig. 6a). Here, it is important to note that we have always used the same amount of catalyst. Thus, the effective amount of photoactive $\mathrm{ZnO}$ is even smaller when $\mathrm{ZnO}-\mathrm{GO}$ is used. The degradation efficiency at 70 minutes of irradiation is $48.6 \%$ for $\mathrm{ZnO}, 65 \%$ for $\mathrm{ZnO}-\mathrm{GO} 1,67 \%$ for $\mathrm{ZnO}-\mathrm{GO} 2$, and $56.3 \%$ for $\mathrm{ZnO}-\mathrm{GO} 3$. The ZnO-GO3 catalyst, synthesized with GO of high oxidation degree, shows less efficiency than the others. This underlines the existence of a certain threshold value beyond which higher oxidation degrees of GO are detrimental for the photocatalytic activity of $\mathrm{ZnO}-\mathrm{GO}$ hybrids. This observation thus provides another proof of the critical role that GO surface chemistry plays in the catalytic activity of ZnO-GOX hybrids.

The photocatalytic degradation of $\mathrm{MB}$ by $\mathrm{ZnO}$, and $\mathrm{ZnO}-\mathrm{GOX}$ materials fitted well to a first-order linear kinetics up to 40 minutes of irradiation (Fig. S6). After this time, the activity of the catalysts declines. The apparent rate constant $\left(\mathrm{k}, \min ^{-1}\right)$, calculated for the 40 first minutes from the slope of the $\ln (\mathrm{A} / \mathrm{Ao})$ vs time curve, increased for the $\mathrm{ZnO}$ GO1 and $\mathrm{ZnO}-\mathrm{GO} 2$ with respect to $\mathrm{ZnO}$, evidencing the improvement of the catalyst efficiency for the degradation of MB when graphene oxide is used.

Analyzing the differences between the $\mathrm{ZnO}-\mathrm{GO} 3$ catalyst and the catalysts obtained with GO1 and GO2, it is clear that the degree of oxidation has a major influence on the final structural characteristics of the hybrid material. Looking back to the structural characterization of the hybrids, we observe that $\mathrm{ZnO}-\mathrm{GO} 3$ catalyst showed the largest increase of the (002) XRD diffraction peak intensity. On the other side, it exhibits the smallest Raman peak at $1100 \mathrm{~cm}^{-1}$. While the higher concentration of oxygen functional 
groups in $\mathrm{GO} 3$ allows a higher level of interfacial interactions with $\mathrm{ZnO}$, resulting in a more compact structure (Fig. S1), concomitantly it obstructs the access to the catalytic centers of $\mathrm{ZnO}$. Consequently, a higher level of interactions, which could be beneficial in other applications, here limits the efficiency of the catalyst, since these apparently affect the active part of the $\mathrm{ZnO}$ catalyst. Thus, it seems clear that graphene oxide has an important role in the improvement of the $\mathrm{ZnO}$ catalysis efficiency and controlling the degree of oxidation is crucial to establish the adequate level of interfacial GO-ZnO interactions for designing efficient catalysts.
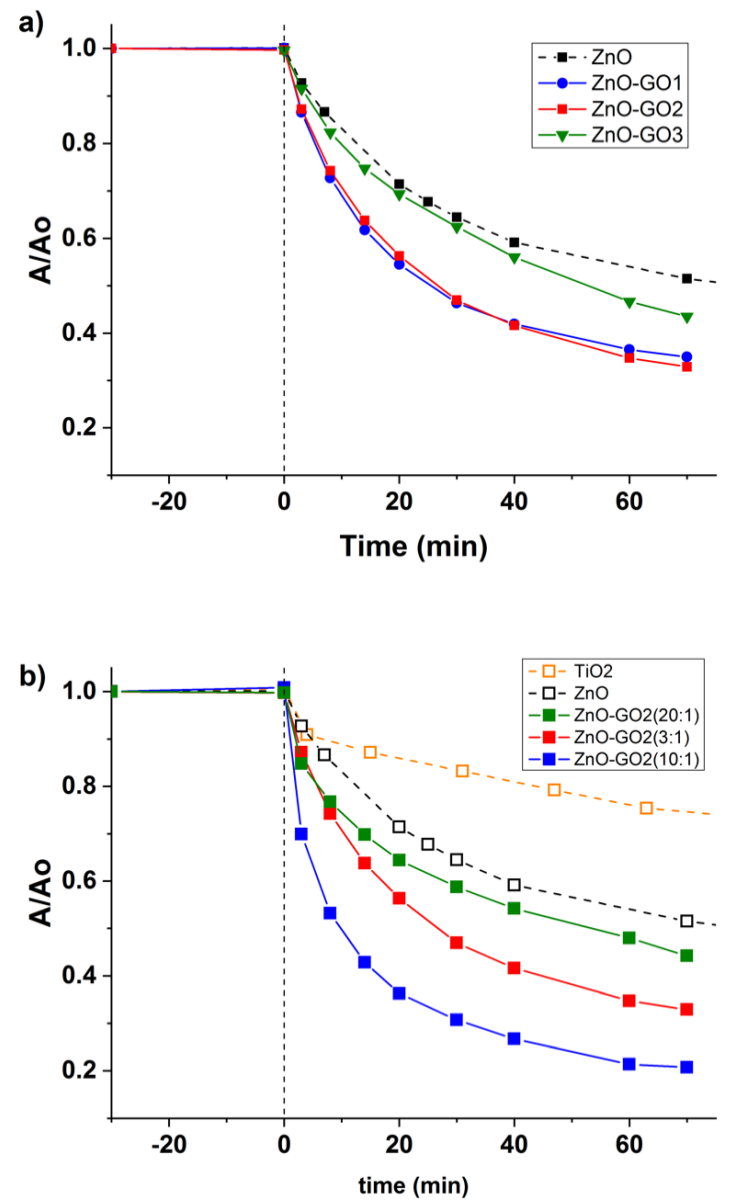

Fig. 6. Photocatalytic degradation of methylene blue $(M B)$ with time under $U V$ light irradiation by a) $\mathrm{ZnO}$ and $\mathrm{ZnO}-\mathrm{GOX}$ catalysts with 3:1 of $\mathrm{ZnO}: G O$ $w: w$ ratio. b) $\mathrm{ZnO}$ and $\mathrm{ZnO}-\mathrm{GO} 2$ catalyst with different $\mathrm{ZnO}: \mathrm{GO}$ w:w ratios 
3.2. Influence of the GO loading on the catalytic activity of the $\mathrm{ZnO}-\mathrm{GO}$ catalysts

One important issue, which notably contributes to improve the activity of the $\mathrm{ZnO}-\mathrm{GO}$ catalysts, is the GO loading. This must be adequately chosen to reach optimal catalytic performance [38]. With this aim, three different $\mathrm{ZnO}: \mathrm{GO}$ ratios $(3: 1,10: 1$ or $20: 1 \mathrm{w} / \mathrm{w})$ for the synthesis of the new set of catalysts were assayed. Here GO2 was chosen as graphene oxide material since the $\mathrm{ZnO}-\mathrm{GO} 2$ catalyst showed the best performance for the degradation of MB.

No differences were observed in the XRD patterns of the $\mathrm{Zn}-\mathrm{GO} 2$ materials with different GO2 loadings (Fig. S3). However, like in the previous section, the relative intensity ratio of the three main diffraction peaks varies when different GO2 loadings are used (Table 3). The $\mathrm{I}_{\mathrm{i}} / \mathrm{I}_{0}$ intensity ratio corresponding to the (100) diffraction peak hardly changes with respect to the $\mathrm{ZnO}$ material, but the corresponding (002) diffraction peak decreases for the hybrids with higher GO2 content, and increases for the catalyst with the lowest $\mathrm{GO} 2$ content $(20: 1 \mathrm{w} / \mathrm{w})$, denoting, once more, that the established interfacial interactions are taking place preferentially through the (002) diffraction plane.

Table 3. Diffraction angles of $\mathrm{ZnO}-\mathrm{GO} 2$ hybrids with different ratios and relative intensity of the peaks.

\begin{tabular}{cccccc}
\hline Miller index & Angle & ZnO & ZnO-GO2 (20:1) & ZnO-GO2 (10:1) & ZnO-GO2 (3:1) \\
\cline { 3 - 6 }$(\mathbf{h}, \mathbf{k}, \mathbf{l})$ & $\mathbf{2 \theta}$ & $\mathrm{I}_{\mathrm{i}} / \mathrm{I}_{0}(\%)$ & $\mathrm{I}_{\mathrm{i}} / \mathrm{I}_{0}(\%)$ & $\mathrm{I}_{\mathrm{i}} / \mathrm{I}_{0}(\%)$ & $\mathrm{I}_{\mathrm{i}} / \mathrm{I}_{0}(\%)$ \\
\cline { 3 - 6 }$(100)$ & 31.8 & 0.65 & 0.64 & 0.66 & 0.67 \\
$(002)$ & 34.5 & 0.55 & 0.59 & 0.50 & 0.51 \\
$(101)$ & 36.3 & 1 & 1 & 1 & 1 \\
\hline
\end{tabular}

Fig. 7 shows the Raman spectra of $\mathrm{ZnO}-\mathrm{GO} 2$ materials with different $\mathrm{GO} 2$ content compared to $\mathrm{GO} 2$ and bare $\mathrm{ZnO}$. Like in Fig. 2, the spectra of $\mathrm{ZnO}-\mathrm{GO} 2$ hybrids exhibit the G- $\left(1598 \mathrm{~cm}^{-1}\right)$ and D- $(1347 \mathrm{~cm}-1)$ bands characteristic of GO2, as well as the 
enhancement of the Raman mode at around $1100 \mathrm{~cm}^{-1}$, indicating the establishment of interactions between $\mathrm{ZnO}$ and GO. Investigating the low frequency region, we observe prominent bands at 332, 438, 580 and $665 \mathrm{~cm}^{-1}$. Among this group, the maximum intensity arises at $438 \mathrm{~cm}^{-1}$. This prominent frequency corresponds to the nonpolar $\mathrm{E}_{2}$ (high) mode, which involves mostly the oxygen atoms of the $\mathrm{ZnO}$ lattice and is characteristic of the wurtzite hexagonal structure of $\mathrm{ZnO}[39,40]$. The relatively higher intensity and sharp $\mathrm{E}_{2}$ (high) mode compared to the other observed peaks demonstrates that the synthesized $\mathrm{ZnO}$ material exhibits good crystallinity [41, 42]. The intensity of this peak significantly decreases when GO2 is added to the catalyst, probably indicating that the new hybrid material has lower crystal quality, and this decrease of crystallinity might be a consequence of the establishment of interactions between $\mathrm{ZnO}$ and graphene. The peak at $580 \mathrm{~cm}^{-1}$ is assigned to the $\mathrm{E}_{1}(\mathrm{LO})$ mode, which is associated with the structural defects in $\mathrm{ZnO}$ and is very sensitive to the inclusion of "oxygen imperfections" in the $\mathrm{ZnO}$ structure $[40,43,44]$. This mode is present in all the materials and it is particularly intense in $\mathrm{ZnO}-\mathrm{GO} 2$ (10:1), evidencing a stronger interaction between $\mathrm{ZnO}$ and oFGs of GO2 in this catalyst. The peaks at 332 and 662 $\mathrm{cm}^{-1}$ are due to multiple-phonon scattering processes $[45,46]$. 

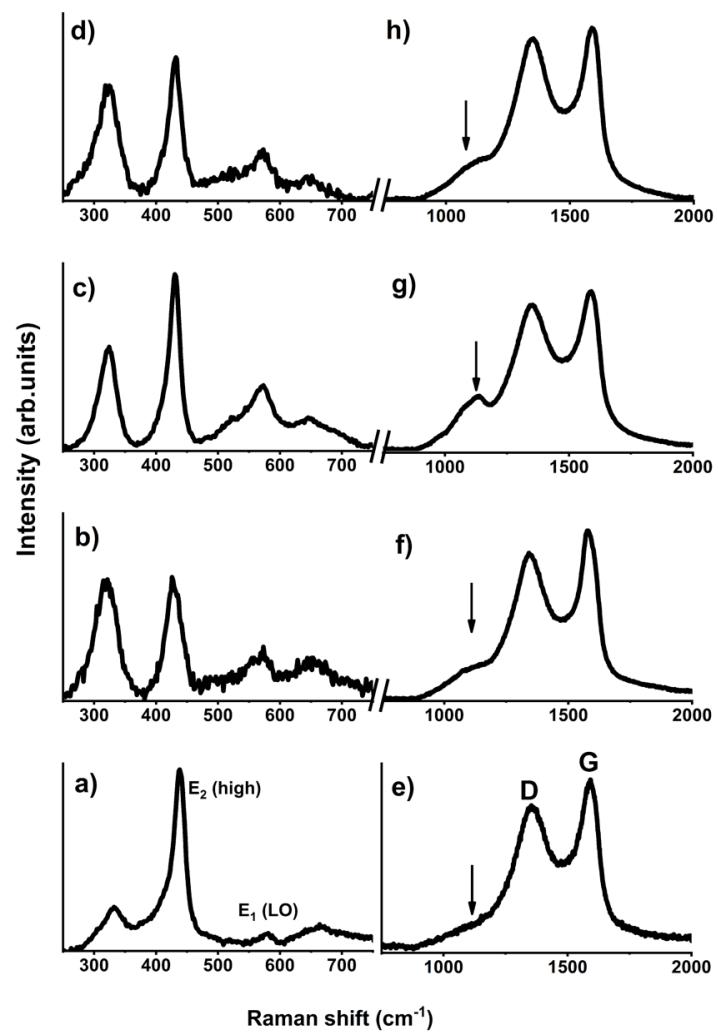

Fig. 7. RAMAN spectra of a) $\mathrm{ZnO}$; $b$, f) $\mathrm{ZnO}-\mathrm{GO} 2(20: 1 \mathrm{w} / \mathrm{w})$; c, g) ZnO-GO2 (10:1 w/w); d, h) ZnO-GO2 (3:1 w/w); e) GO2

FTIR of the ZnO-GO2 hybrids with different loadings (Fig. S5) shows an increase of the $3430 \mathrm{~cm}^{-1} \mathrm{O}-\mathrm{H}$ stretching vibration with the $\mathrm{GO} 2$ content and no signal corresponding to the $\mathrm{C}=\mathrm{O}$ stretching mode at $1734 \mathrm{~cm}^{-1}$ of the $\mathrm{GO} 2$ is observable.

X-ray photoelectron spectroscopy (XPS) analysis was carried out to investigate the surface composition of the prepared catalysts. The C1s spectrum of GO2 (Fig. 8a) was deconvoluted in 5 components at binding energies (BE) of $284.5 \mathrm{eV}$ (I, $\mathrm{C}=\mathrm{C}$ bonds), $285.2 \mathrm{eV}$ (II, C-C bonds), $286.6 \mathrm{eV}$ (III, C-O groups), $287.7 \mathrm{eV}$ (IV, C=O groups), 289 $\mathrm{eV}(\mathrm{V}, \mathrm{COOH}$ groups). The $\mathrm{C} 1 \mathrm{~s}$ spectrum widens for the $\mathrm{Zn}-\mathrm{GO} 2$ hybrids. Thus, two additional peaks have been added on both sides of the spectrum to get a good fitting (grey peaks in Fig 8b,c). The new peak at the lowest BE $(283 \mathrm{eV})$ has been assigned to 
a carbon interaction with $\mathrm{Zn}[47,48]$. And the new peak at the highest $\mathrm{BE}(291 \mathrm{eV})$ has been assigned to the $\pi-\pi^{*}$ shake-up satellite band typical for $\mathrm{sp}^{2}$ hybridized carbon, indicating a partial reduction of $\mathrm{GO}$ during preparation of $\mathrm{ZnO}-\mathrm{GO}$ materials, which might involve a charge transfer between $\mathrm{ZnO}$ and $\mathrm{GO}$. Also, this peak might have some contribution of the interaction between oFGs of $\mathrm{GO}$ and $\mathrm{ZnO}$. The intensity of the peaks in the carbon bonded to oxygen region (peaks III, IV, V) increases for all the $\mathrm{ZnO}-\mathrm{GO} 2$ hybrids, denoting the presence of the oxygen atoms from $\mathrm{ZnO}$ in the crystalline network of GO, and together with the new peak at $283 \mathrm{eV}$ evidencing the establishment of interactions between $\mathrm{ZnO}$ and graphene oxide through the $\mathrm{C}=\mathrm{O}$ group of graphene oxide, as observed in FTIR, i.e. C-O-Zn interactions. The Zn $2 p$ core-level spectra of $\mathrm{ZnO}$ (Fig. 8d) shows the characteristic doublet at BE 1022.3 and $1046.4 \mathrm{eV}$, assigned as $\mathrm{Zn} 2 \mathrm{p}_{3 / 2}$ and $\mathrm{Zn} 2 \mathrm{p}_{1 / 2}$ bands, respectively. The BE separation between both bands is 23 $\mathrm{eV}$, in concordance with the standard reference value for $\mathrm{ZnO}$ [47]. The binding energy of these peaks, their BE separation and the small full width at half maximum (FWHM) values $(2.1 \mathrm{eV})$ indicate that $\mathrm{Zn}$ ions are in +2 oxidation state and, thus, the chemical $\mathrm{Zn}-\mathrm{O}$ bonds are dominant in the $\mathrm{ZnO}$ catalyst. In the $\mathrm{ZnO}-\mathrm{GO} 2$ hybrid, wider FWHM values are observed, $2.6 \mathrm{eV}$ for $\mathrm{ZnO}-\mathrm{GO} 2(3: 1 \mathrm{w} / \mathrm{w})$ and $2.5 \mathrm{eV}$ for $\mathrm{ZnO}-\mathrm{GO} 2$ (10:1 $\mathrm{w} / \mathrm{w})$, indicating the appearance of a $\mathrm{Zn}-\mathrm{O}-\mathrm{C}$ interaction, as it has been seen in the $\mathrm{C} 1 \mathrm{~s}$ spectra. 

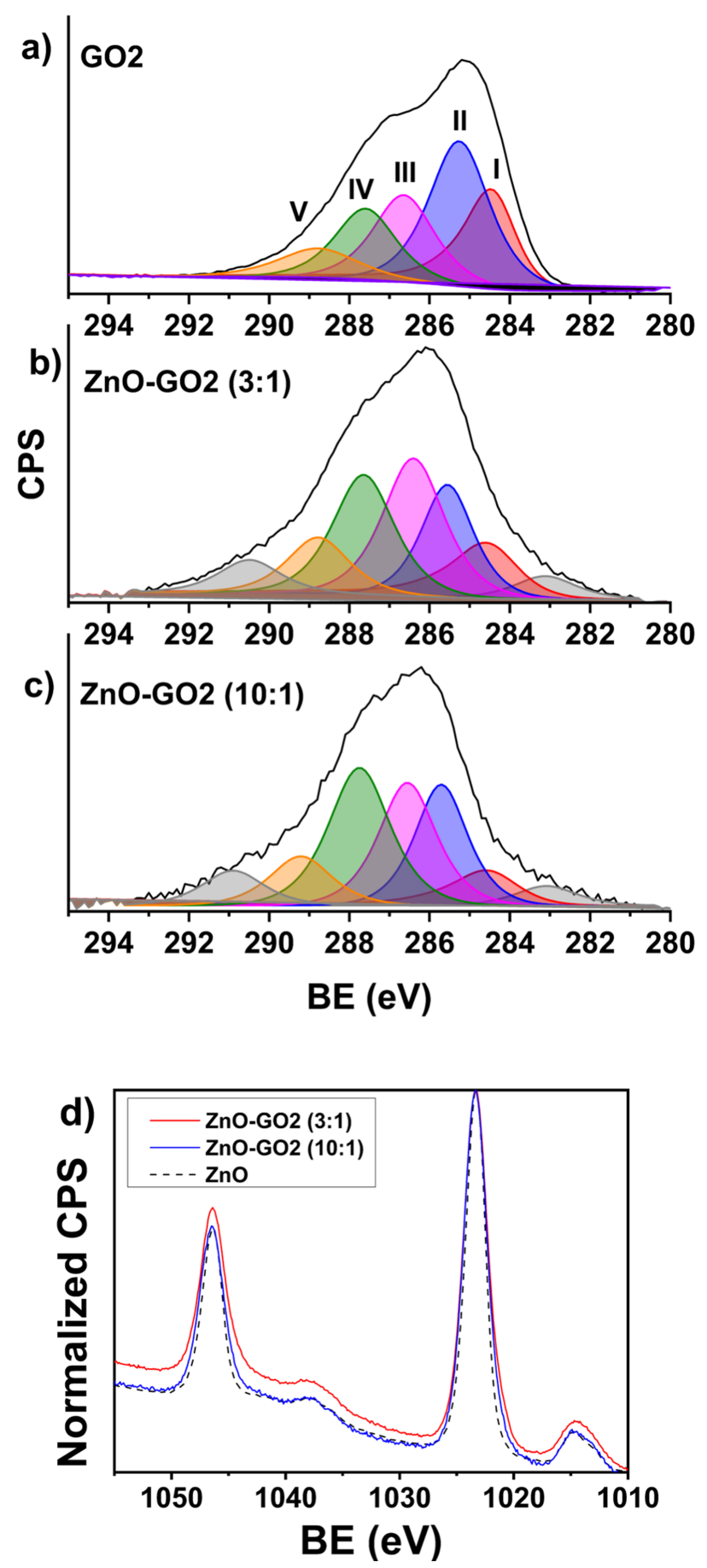

Fig. 8. C1s XPS spectra of a) GO2, b) ZnO-GO2 (3:1 w/w), and c) $\mathrm{ZnO}-\mathrm{GO} 2(10: 1 \mathrm{w} / \mathrm{w})$. d) $\mathrm{Zn} 2 \mathrm{p}$ core level XPS spectra of $\mathrm{ZnO}, \mathrm{ZnO}$ GO2 (3:1 and 10:1 w/w) catalysts

The XPS observations on the $\mathrm{ZnO}-\mathrm{GO} 2$ materials thus are in agreement with the results from optical characterization, whereby the UV-Vis spectra (see Fig. 4) reveal a blueshift of the $\mathrm{ZnO}$ edge and the photoluminescence spectra evidence significant quenching (see 
Fig. 5) indicating the establishment of interface interactions facilitating photo-induced charge transfer between $\mathrm{ZnO}$ and GO.

Next, the three catalysts synthesized with different GO2 loadings were tested in the photocatalytic degradation of methylene blue under UV light irradiation. For comparison, we also examined the photocatalytic activity of the standard reference P25 $\mathrm{TiO}_{2}$ catalyst under the same catalytic conditions (Fig. 6b). After fitting to first-order kinetics, ZnO-GO2 (10:1 w/w) catalyst showed the highest degradation rate $\left(0.03 \mathrm{~m}^{-1}\right)$, which is 1.7 times higher than that of the $\mathrm{ZnO}$ catalyst (Fig S7). Final conversion of $80 \%$ in 70 minutes is obtained with the $\mathrm{ZnO}-\mathrm{GO} 2(10: 1 \mathrm{w} / \mathrm{w})$ catalyst, thus exhibiting a much higher performance than the $\mathrm{P} 25 \mathrm{TiO}_{2}$ catalyst at these conditions.

3.3. Photostability, photoelectrochemical performance and photocatalysis mechanism In order to test the potential up-scalability and stability of our photocatalysts, we carried out photocatalytic degradation experiments in a larger scale. We chose diphenhydramine (DP) as model reaction [43-46] to demonstrate the applicability of our catalyst for the degradation of other organic water pollutants, and we undertook the photodegradation process in a larger container to facilitate the recovery and reuse of the photocatalysts. The conversion outcomes at $60 \mathrm{~min}$ of reaction time and $1 \mathrm{~g} / \mathrm{L}$ catalyst concentration are displayed in Figure 9. It is visible how the results are perfectly consistent with those obtained in by our previous benchmark experiments. Analogous to the case of $\mathrm{MB}$, here the $\mathrm{ZnO}-\mathrm{GO} 2$ catalyst also reveals the best performance for the photodegradation of DP. The recyclability experiments could be easily carried out in contrast to the previous catalyst screening experiments, in which the experimental configuration and smaller amount of catalyst employed, impeded us to efficiently recover it for further reuse. The $\mathrm{ZnO}-\mathrm{GO} 2$ (10:1) catalyst was chosen for this study, 
since it exhibited the best DP conversion (equally as the $\mathrm{ZnO}-\mathrm{GO} 2$ (3:1) catalyst) and the best performance for MB degradation (Fig. 9). Upon the second photocatalytic cycle, the hybrid catalyst experiences a decay in performance, which is indeed a common feature in similar systems. However, the conversion drop is only of about $25 \%$ of the initial value, and afterwards, it seems to become stable, showing nearly the same performance in a third photocatalytic cycle.

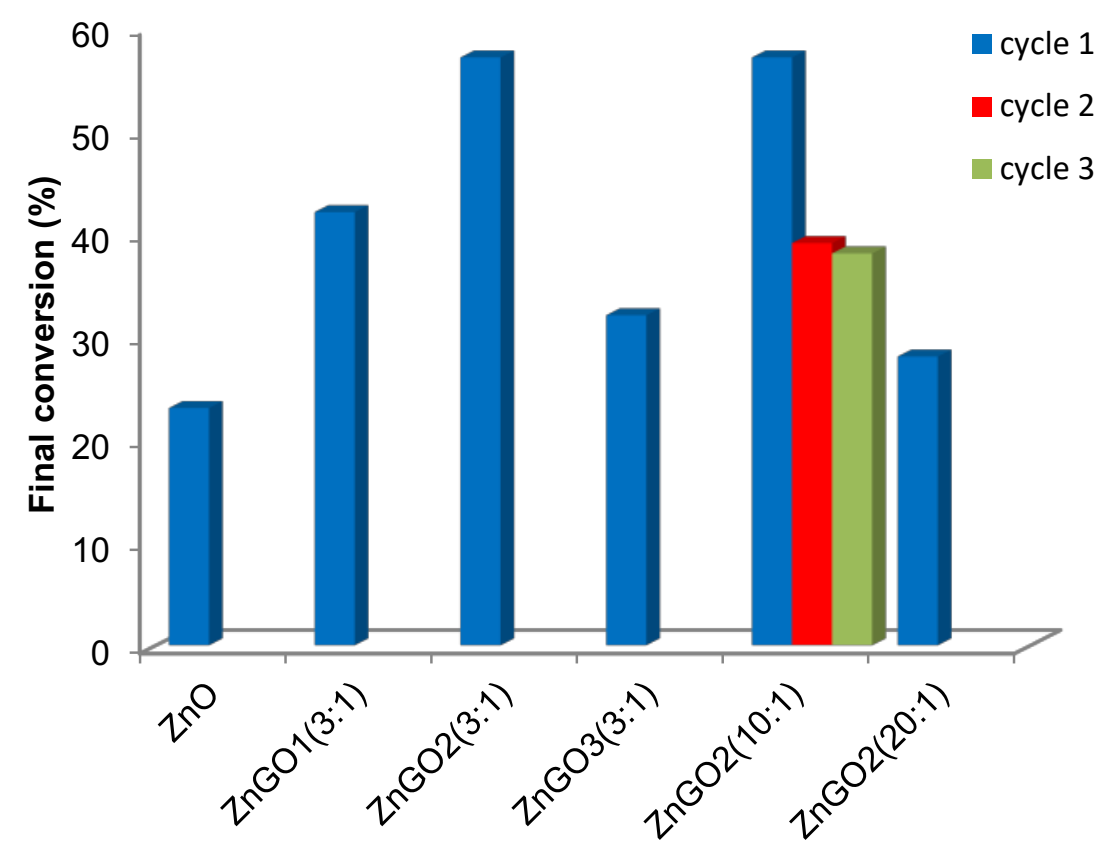

Fig. 9. Photocatalytic performance of $\mathrm{ZnO}, \mathrm{ZnO}-G O X(3: 1), \mathrm{ZnO}-G O 2(10: 1)$ and $\mathrm{ZnO}-\mathrm{GO} 2$ (20:1) materials for the degradation of diphenhydramine and 3 cycles degradation for the $\mathrm{ZnO}-\mathrm{GO} 2$ (10:1) catalyst.

Finally, aiming to evaluate the photocatalytic properties of the $\mathrm{ZnO}-\mathrm{GO} 2(10: 1 \mathrm{w} / \mathrm{w})$ material in other applications, we tested the photoelectrochemical performance of several prepared films (bare $\mathrm{ZnO}, \mathrm{ZnO}-\mathrm{GO} 2, \mathrm{P} 25 \mathrm{TiO}_{2}$ ) for the photoelectrocatalytic oxidation of water. Photocurrents (difference between currents in the dark and under illumination) measured by $\mathrm{CV}$ in the $0.1 \mathrm{M} \mathrm{Na}_{2} \mathrm{SO}_{4}$ electrolyte (Fig. 10a, also see Fig. S8 for original voltammograms) show that the highest activity corresponds to the electrochemically reduced $\mathrm{ZnO}-\mathrm{ECrGO} 2$ hybrid. Additionally, potentiostatic 
photocurrent measurements performed at $0.4 \mathrm{~V}$ (Fig. 10b) reveal that the photocurrent values for $\mathrm{ZnO}-\mathrm{ECrGO} 2$ are 2.25 times higher than for the bare $\mathrm{ZnO}$, and 4 times higher than for the reference catalyst $\mathrm{P} 25 \mathrm{TiO}_{2}$. For this application, electrochemical reduction of the $\mathrm{ZnO}-\mathrm{GO} 2$ hybrid results in a material with higher photocatalytic performance. Please, take into account that we have explored two different reactions: electrochemical water oxidation and photochemical MB degradation. Consequently, the involved processes are different. The original ability of $\mathrm{ZnO}$ material to transfer holes to the solution and/or subsequently, electrons to the FTO electrode decreases with the presence of GO (electrical insulator), yielding a lower current. Upon reduction of GO, the current increases due to the improvement of the conductivity properties of the electrochemical reduced GO. However, independent of the electrochemical reduction step required for the probed system, these findings are in full agreement with the previous results on $\mathrm{MB}$ degradation, whereby $\mathrm{ZnO}-\mathrm{GO} 2$ leads to improved photocatalytic behavior with respect to pristine $\mathrm{ZnO}$ and the reference standard $\mathrm{P} 25$ $\mathrm{TiO}_{2}$. Moreover, they clearly reveal that the photocatalytic enhancement by $\mathrm{ZnO}-\mathrm{GO} 2$ is not limited to $\mathrm{MB}$ or $\mathrm{DP}$ degradation, but of even more general character, is applicable to other types of reactions such as water oxidation. 

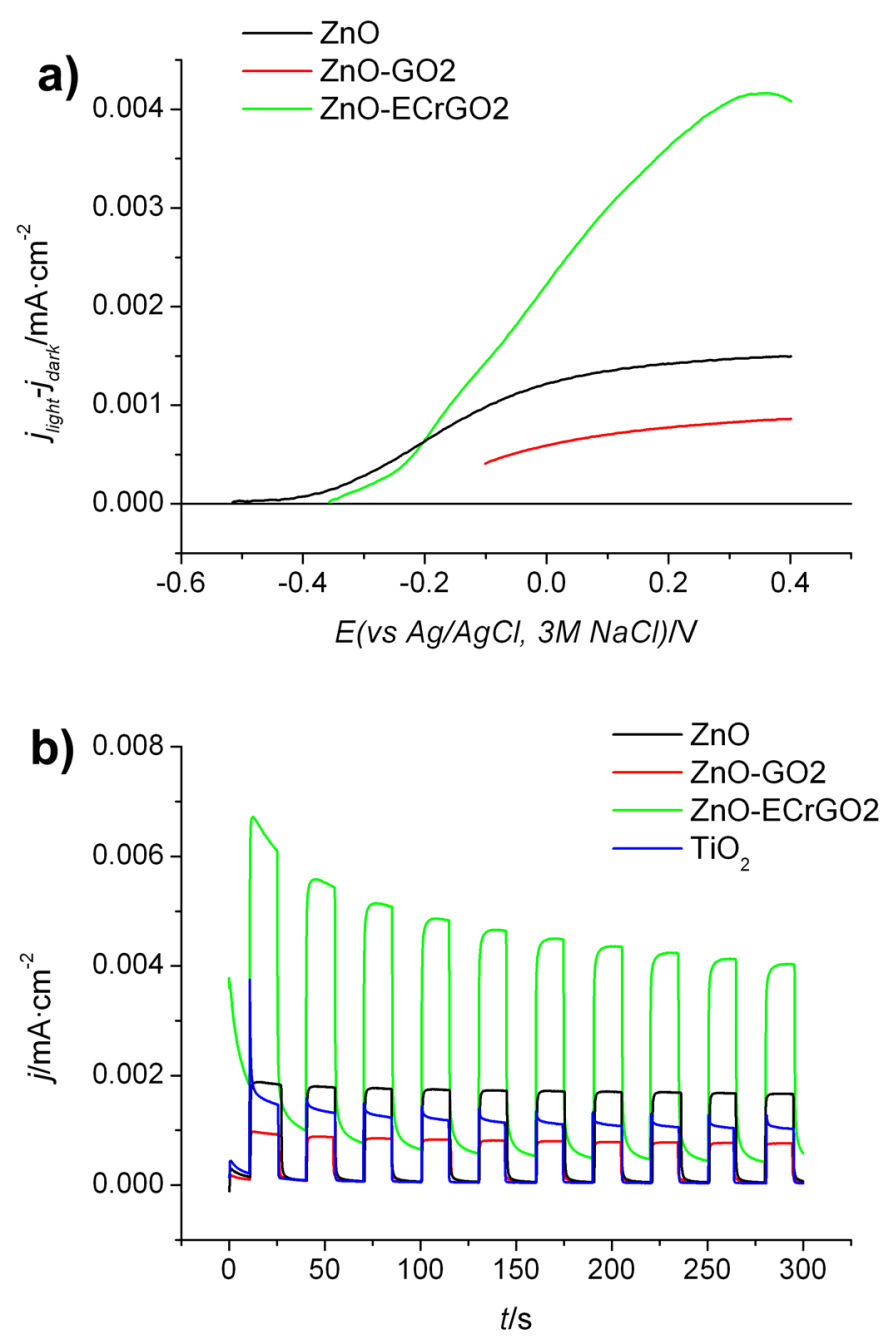

Fig. 10. a) Difference between currents recorded under illumination and in the dark for the different samples in $\mathrm{Na}_{2} \mathrm{SO}_{4}$ and b) potentiostatic on-off samples for water oxidation.

Our experimental findings on the enhancement of photocatalytic and photoelectrochemical activity can be explained at hand of the scheme of energy levels of $\mathrm{ZnO}, \mathrm{GO}$ and $\mathrm{ZnO}-\mathrm{GO}$ shown in Fig. 11. Their values are entirely based on the values obtained from UV-Vis and photoluminescence spectra mentioned in the respective section. In case of the hybrid material it can be seen that trough the establishment of interactions of oxygen functional groups of $\mathrm{GO}$ with those of $\mathrm{ZnO}$ located at its 002 plane, the resulting alignment of energy levels at the $\mathrm{ZnO}-\mathrm{GO}$ interface then facilitates the transfer of photo-excited electrons from $\mathrm{ZnO}$ to $\mathrm{GO}$ states 
of lower energy upon irradiation with UV-light. This reduces the recombination rate of the photo-generated electron-hole pair and promotes oxidation reactions with the analyte or/and its medium via the remaining positive charges (holes) in the valence band of $\mathrm{ZnO}$. In this way, GO contributes to the enhancement of the photocatalytic activity of $\mathrm{ZnO}$. Please note that $\mathrm{GO}$ in itself is not photoactive due to the large energetic separation of the relevant energy levels from its ground-state, a fact which is confirmed as well by the absence of photoactivity in our corresponding blank experiments.

However, this not necessarily assigns GO a purely passive role. On the contrary, the transfer of photo-excited electrons to GO most likely opens additional pathways of more or less complex nature contributing to further improvement of photocatalytic activity of the $\mathrm{ZnO}-\mathrm{GO}$ hybrid material. However, this problematic yet goes beyond the scope of the present study.

(a)

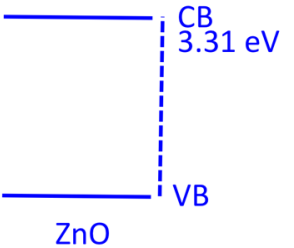

(b)

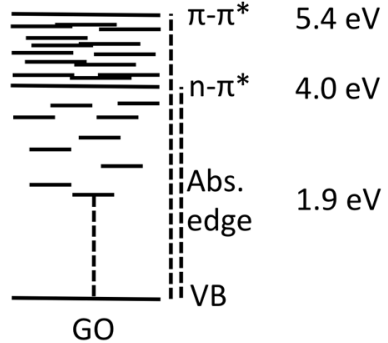

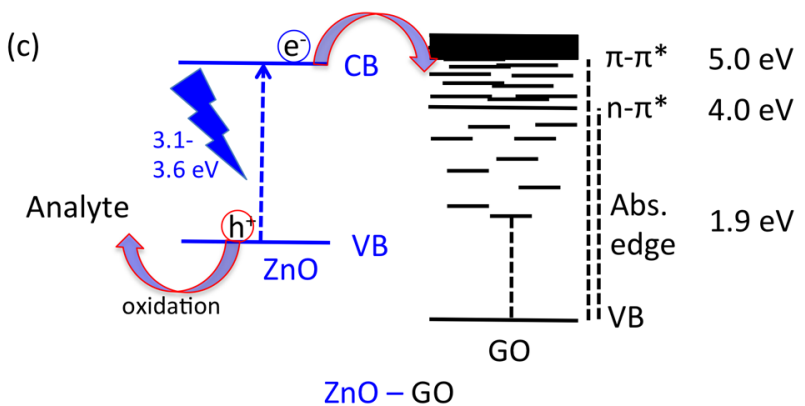

Fig. 11: Schematic diagram of energy levels of (a) $\mathrm{ZnO}$, (b) $\mathrm{GO}$ and (c) $\mathrm{ZnO}$ - $\mathrm{GO}$ displaying valence band (VB), conduction band (CB), absorption edge, $n-\pi^{*}$ and $\pi-\pi^{*}$ levels. ${ }^{1}$ The proposed alignment of the energy levels in $\mathrm{ZnO}-\mathrm{GO}^{2}$ indicates the possibility of photo-induced energy transfer from $\mathrm{ZnO}$ to $\mathrm{GO}$ states of lower energy upon irradiation with UV-light, ${ }^{3}$ thus reducing the $\mathrm{e}^{-}-\mathrm{h}^{+}$recombination rate and enhancing the photoactivity of $\mathrm{ZnO}^{4}$

${ }^{1}$ Energy values taken from UV-Vis absorption and photoluminescence spectroscopy. The lowering of the $\pi-\pi^{*}$ energy level of the $\mathrm{GO}$ component in the $\mathrm{ZnO}-\mathrm{GO}$ by $0.4 \mathrm{eV}$ suggests a (partial) reduction of GO 
enabled by effective interface interactions between oxygen functional groups of $\mathrm{GO}$ and $\mathrm{ZnO}$ at its (002) plane.

${ }^{2}$ The alignment of the energy levels of GO with respect to $\mathrm{ZnO}$ in $\mathrm{ZnO}-\mathrm{GO}$ may vary as a factor of oxygen functional groups of GO and its density of states, as well as the degree of interactions established with the (002) plane of $\mathrm{ZnO}$. Its exact position requires specific measurements beyond the scope of this study.

${ }^{3}$ Excitation energy taken from UV-lamp datasheet.

${ }^{4} \mathrm{GO}$ itself is not photoactive.

With respect to the need for using rGO instead of GO, as claimed in many studies, we would like to point out the following:

As long as favorable interface interactions between $\mathrm{ZnO}$ and $\mathrm{GO}$ are established via the respective oxygen functional groups, catalytic enhancement is achieved through photoinduced charge-transfer processes, as explained above. There is no need for further reduction processes. In fact, the creation of proper interface interactions leads to a significant lowering of the $\pi-\pi^{*}$ transition by $0.4 \mathrm{eV}$, typically for shifts observed upon reduction of GO to $\mathrm{rGO}$, indicative of an enhancement of density of states favoring improved conductivity and mobility.

The accompanying band-alignment taking place at the $\mathrm{ZnO}-\mathrm{GO}$ interface adjusts the GO levels, as a function of the GO oxygen degree, in such a way that electron-transfer from $\mathrm{ZnO}$ to $\mathrm{GO}$ is enabled, most likely to the rather dense states of oxygen functional groups laying underneath the conduction band of GO formed by its $\pi^{*}$ states. Please note that electron-transfer to the conduction band of GO itself is highly unlikely, as suggested by our photoelectrochemical experiments (see below).

Moreover, the fact that our posterior thermal reduction processes of the $\mathrm{ZnO}-\mathrm{GO}$ hybrids did not contribute to further improvement of the photocatalytic activity (on the contrary, lower activities were observed), underlines that in our $\mathrm{ZnO}-\mathrm{GO}$ hybrids the photocatalytic process is governed by the established interface interactions. Upon 
thermal processes, reduction from GO to more conductive rGO may be achieved, however, we speculate that this is on cost of the established GO-ZnO interface.

In addition, the direct use of rGO to obtain $\mathrm{ZnO}-\mathrm{GO}$ hybrids is not fruitful through the low dispersibility of rGO in water. This did not result in the formation of homogeneous hybrid materials with photocatalytic properties.

Finally, in what concerns the photoelectrochemical experiment: Photoelectrochemistry relies on the availability of conductive electrode materials. The observation that only by applying an electrochemical reduction step to the $\mathrm{ZnO}-\mathrm{GO}$ material a photoelectrochemical active material with superior properties is achieved, suggests that photo-generated electrons are not transferred to the GO conduction band, but rather to the underlying dense states formed by the oxygen functional groups. The irreversible electrochemical reduction step, then transforms these states into rather continuous network of states allowing for the required conductivity. (Please note that if the photogenerated electron would be transferred to the conduction band of GO, no further reduction process would be required to obtain a conductive electrode material). At this point it is important to underline that all this applies to $\mathrm{ZnO}-\mathrm{GO}$ materials in which effective interface interactions are established via the functional oxygen groups of GO. However, in $\mathrm{ZnO}-\mathrm{GO}$ materials where this is not achieved (e.g. due to different synthesis conditions, or non-appropriate oxidation degrees of GO), the presence of more conductive rGO may open additional pathways of direct interactions with the analyte/medium thus enhancing the overall photocatalytic activity in these types of hybrid materials. These observations are also in agreement with our former studies on $\mathrm{TiO}_{2}-\mathrm{GO}$ hybrid materials $[31,32]$. We therefore believe that establishing appropriate interface interactions through adequate synthesis routes and oxidation degrees of GO is 
key to achieve enhanced photocatalytic performance without the need for applying additional reduction steps.

\section{Conclusions}

Using graphene oxide with distinct and well-defined oxidation degree for the preparation of $\mathrm{ZnO}-\mathrm{GO}$ hybrid materials, we demonstrated that the surface chemistry of graphene oxide is key for establishing interface interactions with zinc oxide, which favorably contribute to improve its photocatalytic and photoelectrochemical activity. We showed that the interactions are established between $\mathrm{C}=\mathrm{O}$ groups of $\mathrm{GO}$ and the (002) crystalline plane of $\mathrm{ZnO}$. The resulting alignment of energy levels at the $\mathrm{ZnO}-\mathrm{GO}$ interface then facilitates the transfer of photo-excited electrons form $\mathrm{ZnO}$ to GO. The remaining positive charges (holes) located at the valence band of $\mathrm{ZnO}$ then easily can promote oxidation reactions with the analyte or/and the medium in which it is dissolved. photo-induced charge transfer interactions. In this way GO reduces the recombination rate of the photogenerated electron-hole pair and thus contributes to the enhancement of the photocatatytic activity of $\mathrm{ZnO}$. Most favorable conditions are achieved for an intermediate oxidation degree of GO, while the GO loading fraction needs to be adjusted to ensure the availability of sufficient number of catalytically active $\mathrm{ZnO}$ sites. Based on these findings, an optimized $\mathrm{ZnO}-\mathrm{GO}$ hybrid catalyst was prepared and employed in the catalytic degradation reaction of methylene blue revealing conversion rates of $80 \%$ within a time of 70 minutes obtained at a catalyst concentration of only $0.045 \mathrm{mg} / \mathrm{mL}$.

Moreover, the $\mathrm{ZnO}-\mathrm{GO}$ catalyst demonstrated high performance and recyclability for the degradation of diphenhydramine and good performance for the 
photoelectrochemical oxidation of water. These results are of general character and extendable to other reactions or semiconducting metal oxides used in photocatalytic or photoelectrochemical applications.

\section{Acknowledgements}

This work was financially supported by the Spanish MINEICO (project grant ENE 201679282-C5-1-R), Gobierno de Aragón (Grupo Reconocido DGA T03_17R), and associated EU Regional Development Funds. This work has been also partially supported by Project POCI-01-0145-FEDER-006984 - Associate Laboratory LSRE-LCM funded by European Regional Development Fund (ERDF) through COMPETE2020-Programa Operacional Competitividade e Internacionalização (POCI) - and by national funds through FCT-Fundação para a Ciência e a Tecnologia. S. Víctor-Roman acknowledges Spanish MINEICO for her PhD grant (BES2014-068727 and associated EU Social Funds) and for her research stay scholarship in Portugal (EEBB-I-18-12785). J.M. González-Domínguez acknowledges Spanish Ministry of Science, Innovation and Universities for his 'Juan de la Cierva - Incorporación' grant.

\section{References}

[1] Q. Xiang, J. Yu, M. Jaroniec, Graphene-based semiconductor photocatalysts, Chemical Society Reviews, 41 (2012) 782-796.

[2] A.J. Paulista Neto, E.E. Fileti, Elucidating the amphiphilic character of graphene oxide, Physical Chemistry Chemical Physics, 20 (2018) 9507-9515.

[3] W.S. Hummers, R.E. Offeman, Preparation of Graphitic Oxide, Journal of the American Chemical Society, 80 (1958) 1339.

[4] D.C. Marcano, D.V. Kosynkin, J.M. Berlin, A. Sinitskii, Z. Sun, A. Slesarev, L.B. Alemany, W. Lu, J.M. Tour, Improved Synthesis of Graphene Oxide, ACS Nano, 4 (2010) 4806-4814.

[5] D.R. Dreyer, S. Park, C.W. Bielawski, R.S. Ruoff, The chemistry of graphene oxide, Chemical Society Reviews, 39 (2010) 228-240.

[6] J.D. Nunez, A.M. Benito, S. Rouziere, P. Launois, R. Arenal, P.M. Ajayan, W.K. Maser, Graphene oxide-carbon nanotube hybrid assemblies: cooperatively strengthened $\mathrm{OH}$ center dot center dot center dot $\mathrm{O}=\mathrm{C}$ hydrogen bonds and the removal of chemisorbed water, Chemical Science, 8 (2017) 4987-4995. 
[7] A. Tararan, A. Zobelli, A.M. Benito, W.K. Maser, O. Stéphan, Revisiting Graphene Oxide Chemistry via Spatially-Resolved Electron Energy Loss Spectroscopy, Chemistry of Materials, 28 (2016) 3741-3748.

[8] D. Li, M.B. Müller, S. Gilje, R.B. Kaner, G.G. Wallace, Processable aqueous dispersions of graphene nanosheets, Nature Nanotechnology, 3 (2008) 101-105.

[9] O.C. Compton, S.W. Cranford, K.W. Putz, Z. An, L.C. Brinson, M.J. Buehler, S.T. Nguyen, Tuning the Mechanical Properties of Graphene Oxide Paper and Its Associated Polymer Nanocomposites by Controlling Cooperative Intersheet Hydrogen Bonding, Acs Nano, 6 (2012) 2008-2019.

[10] F. Bonaccorso, L. Colombo, G.H. Yu, M. Stoller, V. Tozzini, A.C. Ferrari, R.S. Ruoff, V. Pellegrini, Graphene, related two-dimensional crystals, and hybrid systems for energy conversion and storage, Science, 347 (2015).

[11] R. Raccichini, A. Varzi, S. Passerini, B. Scrosati, The role of graphene for electrochemical energy storage, Nature Materials, 14 (2015) 271-279.

[12] Y. Liu, Y. Hu, M. Zhou, H. Qian, X. Hu, Microwave-assisted non-aqueous route to deposit well-dispersed $\mathrm{ZnO}$ nanocrystals on reduced graphene oxide sheets with improved photoactivity for the decolorization of dyes under visible light, Applied Catalysis B-Environmental, 125 (2012) 425-431.

[13] K. Huang, Y.H. Li, S. Lin, C. Liang, H. Wang, C.X. Ye, Y.J. Wang, R. Zhang, D.Y. Fan, H.J. Yang, Y.G. Wang, M. Lei, A facile route to reduced graphene oxide-zinc oxide nanorod composites with enhanced photocatalytic activity, Powder Technology, 257 (2014) 113-119.

[14] D. Fu, G. Han, Y. Chang, J. Dong, The synthesis and properties of ZnO-graphene nano hybrid for photodegradation of organic pollutant in water, Materials Chemistry and Physics, 132 (2012) 673-681.

[15] C.B. Ong, L.Y. Ng, A.W. Mohammad, A review of ZnO nanoparticles as solar photocatalysts: Synthesis, mechanisms and applications, Renewable \& Sustainable Energy Reviews, 81 (2018) 536-551.

[16] G. Du, X. Wang, L. Zhang, Y. Feng, Y. Li, Controllable synthesis of different ZnO architectures decorated reduced graphene oxide nanocomposites, Materials Letters, 96 (2013) 128-130.

[17] B. Li, H. Cao, ZnO@graphene composite with enhanced performance for the removal of dye from water, Journal of Materials Chemistry, 21 (2011) 3346-3349.

[18] N.P. Herring, S.H. Almahoudi, C.R. Olson, M.S. El-Shall, Enhanced photocatalytic activity of ZnO-graphene nanocomposites prepared by microwave synthesis, Journal of Nanoparticle Research, 14 (2012).

[19] M. Ahmad, E. Ahmed, Z.L. Hong, J.F. Xu, N.R. Khalid, A. Elhissi, W. Ahmed, A facile one-step approach to synthesizing $\mathrm{ZnO} / g$ raphene composites for enhanced degradation of methylene blue under visible light, Applied Surface Science, 274 (2013) 273-281.

[20] T. Lv, L. Pan, X. Liu, Z. Sun, Enhanced photocatalytic degradation of methylene blue by ZnOreduced graphene oxide-carbon nanotube composites synthesized via microwave-assisted reaction, Catalysis Science \& Technology, 2 (2012) 2297-2301.

[21] C. Zhang, J. Zhang, Y. Su, M. Xu, Z. Yang, Y. Zhang, ZnO nanowire/reduced graphene oxide nanocomposites for significantly enhanced photocatalytic degradation of Rhodamine 6G, Physica E-Low-Dimensional Systems \& Nanostructures, 56 (2014) 251-255.

[22] B. Li, T. Liu, Y. Wang, Z. Wang, ZnO/graphene-oxide nanocomposite with remarkably enhanced visible-light-driven photocatalytic performance, Journal of Colloid and Interface Science, 377 (2012) 114-121.

[23] T.-F. Yeh, J. Cihlar, C.-Y. Chang, C. Cheng, H. Teng, Roles of graphene oxide in photocatalytic water splitting, Materials Today, 16 (2013) 78-84.

[24] S.Z. Liu, H.Q. Sun, S.M. Liu, S.B. Wang, Graphene facilitated visible light photodegradation of methylene blue over titanium dioxide photocatalysts, Chemical Engineering Journal, 214 (2013) 298-303. 
[25] X.Y. Pan, M.Q. Yang, Y.J. Xu, Morphology control, defect engineering and photoactivity tuning of $\mathrm{ZnO}$ crystals by graphene oxide - a unique 2D macromolecular surfactant, Physical Chemistry Chemical Physics, 16 (2014) 5589-5599.

[26] M.-Q. Yang, N. Zhang, M. Pagliaro, Y.-J. Xu, Artificial photosynthesis over graphenesemiconductor composites. Are we getting better?, Chemical Society Reviews, 43 (2014) 82408254.

[27] N. Zhang, M.-Q. Yang, S. Liu, Y. Sun, Y.-J. Xu, Waltzing with the Versatile Platform of Graphene to Synthesize Composite Photocatalysts, Chemical Reviews, 115 (2015) 10307-10377. [28] B. Weng, Y.-J. Xu, What if the Electrical Conductivity of Graphene Is Significantly Deteriorated for the Graphene-Semiconductor Composite-Based Photocatalysis?, Acs Applied Materials \& Interfaces, 7 (2015) 27948-27958.

[29] F. Wang, Y. Zhou, X. Pan, B. Lu, J. Huang, Z. Ye, Enhanced photocatalytic properties of ZnO nanorods by electrostatic self-assembly with reduced graphene oxide, Physical Chemistry Chemical Physics, 20 (2018) 6959-6969.

[30] W. Mei, M. Lin, C. Chen, Y. Yan, L. Lin, Low-temperature synthesis and sunlight-catalytic performance of flower-like hierarchical graphene oxide/ZnO macrosphere, Journal of Nanoparticle Research, 20 (2018).

[31] L.M. Pastrana-Martinez, S. Morales-Torres, V. Likodimos, P. Falaras, J.L. Figueiredo, J.L. Faria, A.M.T. Silva, Role of oxygen functionalities on the synthesis of photocatalytically active graphene-TiO2 composites, Applied Catalysis B-Environmental, 158 (2014) 329-340.

[32] L.M. Pastrana-Martinez, S. Morales-Torres, V. Likodimos, J.L. Figueiredo, J.L. Faria, P. Falaras, A.M.T. Silva, Advanced nanostructured photocatalysts based on reduced graphene oxide-TiO2 composites for degradation of diphenhydramine pharmaceutical and methyl orange dye, Applied Catalysis B-Environmental, 123 (2012) 241-256.

[33] C. Valles, J. David Nunez, A.M. Benito, W.K. Maser, Flexible conductive graphene paper obtained by direct and gentle annealing of graphene oxide paper, Carbon, 50 (2012) 835-844.

[34] S. Khanchandani, S. Kundu, A. Patra, A.K. Ganguli, Band Gap Tuning of ZnO/In2S3 Core/Shell Nanorod Arrays for Enhanced Visible-Light-Driven Photocatalysis, Journal of Physical Chemistry C, 117 (2013) 5558-5567.

[35] Ü. Özgür, Y.I. Alivov, C. Liu, A. Teke, M.A. Reshchikov, S. Doğan, V. Avrutin, S.J. Cho, H. Morko, A comprehensive review of $\mathrm{ZnO}$ materials and devices, Journal of Applied Physics, 98 (2005) 1-103.

[36] A.C. Ferrari, J. Robertson, Interpretation of Raman spectra of disordered and amorphous carbon, Phys. Rev. B, 61 (2000) 14095-14107.

[37] G. Xiong, U. Pal, J.G. Serrano, K.B. Ucer, R.T. Williams, Photoluminescence and FTIR study of ZnO nanoparticles: The impurity and defect perspective, Physica Status Solidi (C) Current Topics in Solid State Physics, 3 (2006) 3577-3581.

[38] X. Zhou, T. Shi, H. Zhou, Hydrothermal preparation of ZnO-reduced graphene oxide hybrid with high performance in photocatalytic degradation, Applied Surface Science, 258 (2012) 62046211.

[39] M. Gautam, M. Verma, G. Misra, Structural and Optical Properties of ZnO Nanocrystals, Journal of Biomedical Nanotechnology, 7 (2011) 161-162.

[40] J.M. Calleja, M. Cardona, RESONANT RAMAN-SCATTERING IN ZNO, Physical Review B, 16 (1977) 3753-3761.

[41] R. Zhang, P.-G. Yin, N. Wang, L. Guo, Photoluminescence and Raman scattering of ZnO nanorods, Solid State Sciences, 11 (2009) 865-869.

[42] C. Li, J. Zhang, H. Yu, L. Zhang, Raman and Photoluminescence Properties of ZnO Nanorods with Wurtzite Structure, Progress in Functional Materials, 538 (2013) 50-53.

[43] P.B. Samanta, AK, Chemical growth of hexagonal zinc oxide nanorods and their optical properties, Applied Nanoscience, 2 (2012) 111-117. 
[44] P.K. Sharma, M. Kumar, A.C. Pandey, Green luminescent ZnO:Cu2+ nanoparticles for their applications in white-light generation from UV LEDs, Journal of Nanoparticle Research, 13 (2011) 1629-1637.

[45] X.Y. Ye, Y.M. Zhou, Y.Q. Sun, J. Chen, Z.Q. Wang, Preparation and characterization of Ag/ZnO composites via a simple hydrothermal route, Journal of Nanoparticle Research, 11 (2009) 11591166.

[46] Z.Y. Zhan, L.X. Zheng, Y.Z. Pan, G.Z. Sun, L. Li, Self-powered, visible-light photodetector based on thermally reduced graphene oxide- $\mathrm{ZnO}(\mathrm{rGO}-\mathrm{ZnO})$ hybrid nanostructure, Journal of Materials Chemistry, 22 (2012) 2589-2595.

[47] C.R. Wagner, WM; Davis, LE, Handbook of X-ray Photoelectron Spectroscopy, Perkin-Elmer, Co., Minnesota, 1979.

[48] D.K. Mishra, J. Mohapatra, M.K. Sharma, R. Chattarjee, S.K. Singh, S. Varma, S.N. Behera, S.K. Nayak, P. Entel, Carbon doped ZnO: Synthesis, characterization and interpretation, Journal of Magnetism and Magnetic Materials, 329 (2013) 146-152. 